\title{
Compositional Abstraction-based Synthesis for Continuous-Time Stochastic Hybrid Systems ${ }^{\underline{4}}$
}

\author{
Ameneh Nejati ${ }^{\mathrm{a}}$, Sadegh Soudjani ${ }^{\mathrm{b}}$, Majid Zamani ${ }^{\mathrm{c}, \mathrm{d}}$ \\ ${ }^{a}$ Department of Electrical Engineering, Technical University of Munich, Germany \\ ${ }^{b}$ School of Computing, Newcastle University, United Kingdom \\ ${ }^{c}$ Department of Computer Science, University of Colorado Boulder, USA \\ ${ }^{d}$ Department of Computer Science, LMU Munich, Germany
}

\begin{abstract}
In this paper, we propose a compositional framework for the construction of discrete-time finite abstractions, also known as finite Markov decision processes, from continuous-time stochastic hybrid systems by quantifying the distance between their outputs in a probabilistic setting. The proposed scheme is based on the notion of stochastic simulation functions, which is used to relate continuoustime stochastic systems with their discrete-time counterparts. Accordingly, one can employ discrete-time abstract systems as substitutions of the continuoustime ones in the controller design process with guaranteed error bounds on their output trajectories. To this end, we first derive sufficient small-gain type conditions for the compositional quantification of the probabilistic distance between the interconnection of original continuous-time stochastic hybrid systems and their discrete-time (finite or infinite) abstractions. We then construct finite abstractions together with their corresponding stochastic simulation functions for a particular class of nonlinear stochastic hybrid systems having some stability property. We illustrate the effectiveness of the proposed results by applying our approaches to the temperature regulation in a circular building and constructing compositionally a discrete-time abstraction from its original continuous-time
\end{abstract}

\footnotetext{
This work was supported in part by the H2020 ERC Starting Grant AutoCPS (grant agreement No. 804639) and the German Research Foundation (DFG) through the grant ZA 873/1-1.

* Corresponding author

Email address: amy.nejati@tum.de (Ameneh Nejati)

Preprint submitted to European Journal of Control
} 
dynamics in a network containing 1000 rooms. We employ the constructed discrete-time abstractions as substitutes to compositionally synthesize policies regulating the temperature of each room for a bounded time horizon.

Keywords: Compositional Abstraction-based Synthesis, Continuous-Time Stochastic Hybrid Systems, Small-Gain Conditions, Finite Markov Decision Processes, Formal Synthesis.

\section{Introduction}

Motivations. Controller design for continuous-time stochastic hybrid systems to enforce complex logical properties such as those expressed as linear temporal logic formulae [1] has been inherently a challenging task due to con-

5 tinuous state sets. In order to overcome this problem, one promising solution is to first abstract the original system by a simpler one possibly discrete in time and finite in space, design a controller for the abstract system, and then refine the controller back to the concrete (original) system via an interface map, by providing guaranteed error bounds on the distance between their output trajectories. Construction of finite abstractions was introduced and used over the last decade to alleviate the complexity of controller synthesis problems. In finite abstractions, each discrete state and input respectively correspond to an aggregate of continuous states and inputs of the original system. Since the abstractions are finite, the algorithmic machineries from computer science [2] are applicable to synthesize controllers for concrete systems.

Unfortunately, construction of finite abstractions for complex systems in a monolithic way suffers from the so-called curse of dimensionality: the complexity grows exponentially with the dimension of the state set. To reduce this issue, one promising approach is to consider the large-scale stochastic system as an 20 interconnected system composed of several smaller subsystems, and perform the abstraction in a compositional manner.

Related Literature. In the past few years, new results obtained for the construction of finite abstractions of continuous-time stochastic systems. Reach- 
ability analysis for continuous-time stochastic systems is presented in [3], which constructs finite-state Markov chain with provable error bounds. Finite bisimilar abstractions for incrementally stable stochastic control systems without discrete dynamics are presented in [4. Abstraction techniques for randomly switched stochastic systems and incrementally stable stochastic switched systems are discussed in [5] and [6], respectively. Although original systems in [4, 6, 5] are in the stochastic settings, their proposed abstractions are finite labeled transition systems whereas in this work we consider finite Markov decision processes (MDPs) as our finite abstractions. An approximation framework for constructing infinite abstractions for jump-diffusion processes is proposed in [7]. Compositional construction of infinite abstractions is discussed in [8] using small-gain 35 type conditions.

There have been also several results for discrete-time stochastic systems with continuous-state spaces. Existing results include finite abstractions for formal synthesis of discrete-time stochastic hybrid systems [9]. A sequential and adaptive girdding approach is proposed in [10, 11] with dedicated tools FAUST ${ }^{2}[12$ and StocHy [13]. Furthermore, formal abstraction-based policy synthesis is discussed in [14, 15]. Compositional construction of infinite abstractions (model order reductions) using small-gain type conditions is presented in [16]. Compositional construction of finite abstractions for discrete-time stochastic control systems is presented in [17] and [18] using respectively dynamic Bayesian networks and dissipativity properties of subsystems and their abstractions. A new notion of disturbance bisimulation relation is proposed in [19] for compositional construction of finite abstractions. Recently, a notion of approximate simulation relation for stochastic systems is proposed in [20] that is based on lifting probabilistic evolution of systems to a coupled space. This notion is extended 50 in 21] for compositional abstraction-based synthesis of general MDPs. This notion enables using both model order reduction and space discretization in a unified framework.

There have been several results on the construction of finite abstractions for non-stochastic systems. Construction of symbolic models for incrementally 
stable nonlinear control systems is proposed in [22]. Extension of the results to switched systems in given in [23]. Abstraction of nonlinear control systems without stability assumptions is proposed in [24]. A new approach for hierarchical control based on a quantitative version of simulation relations is proposed in [25] which is based on constructing infinite abstractions (reduced order models).

In the context of stability analysis of large-scale non-stochastic systems via small-gain conditions, several results have been proposed in the past few years. Stability verification of large-scale power systems with delay using the smallgain theorem is proposed in [26]. Stability analysis of hybrid systems described as feedback interconnections of smaller subsystems is proposed in [27] using a Lyapunov-based small-gain framework. Construction of strong and weak Lyapunov functions for a feedback connection of two hybrid systems satisfying certain Lyapunov stability assumptions together with a small-gain condition is presented in [28]. Stability verification of nonlinear control systems under intermittent information via the small-gain theorem is studied in [29, 30].

Contributions. Our main contribution in this paper is to propose for the first time a compositional framework for the construction of discrete-time finitespace MDPs from continuous-time stochastic hybrid systems. We leverage sufficient small-gain conditions to provide the compositionality results which rely on a relation between the continuous-time subsystems and their discrete-time 75 counterparts by employing a notion of stochastic simulation functions. This type of relations enables us to compute a probability bound between the interconnection of continuous-time concrete subsystems and that of their discrete-time (in)finite abstractions. We show that if the original stochastic hybrid system has a stability property, the probability bound associated with the non-stochastic abstractions is less conservative than that of stochastic ones.

In this respect, we first compositionally quantify the distance between the interconnection of continuous-time stochastic hybrid subsystems and that of their discrete-time (finite or infinite) abstractions in a probabilistic setting. We then construct finite abstractions together with their corresponding stochastic simulation functions for a particular class of nonlinear stochastic hybrid systems. 
To illustrate the effectiveness of the proposed results, we apply our approaches to a temperature regulation in a circular building (presented as a running example) and construct compositionally a discrete-time abstraction of a network containing 1000 rooms. We employ the constructed discrete-time abstractions 90 as substitutes to compositionally synthesize policies regulating the temperature of each room for a bounded time horizon.

Recent Work. A limited subset of the provided results in this paper has been recently presented in [31. Our approach here differs from the one in [31] in three main directions. First and foremost, we provide here a compositional

${ }_{95}$ framework for the construction of finite MDPs for networks of continuous-time stochastic hybrid systems, whereas the proposed results in [31 only deal with a single system. In this regard, we propose a max small-gain condition in which the overall error is completely independent of the size of the network, and is computed only based on the maximum errors associated to subsystems. Second, we enlarge the class of systems to continuous-time stochastic hybrid ones, while the results in [31] only deal with stochastic control systems. As our third main contribution, we propose a construction framework of finite MDPs for a particular class of nonlinear stochastic hybrid systems by adding nonlinear terms together with Poisson processes to the dynamics, whereas the results in 31 only handle a class of linear affine systems. In addition, we provide the proofs of all statements here which were omitted in [31.

Organization. The rest of the paper is organized as follows. Section 2 gives mathematical preliminaries and notations, and also the formal definition of continuous-time stochastic hybrid systems. In Section 3 , we first introduce a notion of stochastic pseudo-simulation functions for the stochastic hybrid subsystems with internal inputs, and then define the stochastic simulation functions for the interconnected systems without internal inputs. Section 4 contains the main compositionality results of the paper. In Section 5, we provide a construction framework of finite MDPs for a particular class of nonlinear stochastic hybrid systems. 


\section{Preliminaries and Model Classes}

\subsection{Preliminaries}

We consider a probability space $\left(\Omega, \mathcal{F}_{\Omega}, \mathbb{P}_{\Omega}\right)$, where $\Omega$ is the sample space, $\mathcal{F}_{\Omega}$ is a sigma-algebra on $\Omega$ comprising subsets of $\Omega$ as events, and $\mathbb{P}_{\Omega}$ is a probability measure that assigns probabilities to events. We assume that random variables in this paper are measurable functions of the form $X:\left(\Omega, \mathcal{F}_{\Omega}\right) \rightarrow\left(\mathcal{S}_{X}, \mathcal{F}_{X}\right)$. Any random variable $X$ induces a probability measure on its space $\left(\mathcal{S}_{X}, \mathcal{F}_{X}\right)$ as $\operatorname{Prob}\{\mathcal{A}\}=\mathbb{P}_{\Omega}\left\{X^{-1}(\mathcal{A})\right\}$ for any $\mathcal{A} \in \mathcal{F}_{X}$. We often directly discuss the probability measure on $\left(\mathcal{S}_{X}, \mathcal{F}_{X}\right)$ without explicitly mentioning the underlying probability space and the function $X$ itself.

A topological space $\mathcal{S}$ is called a Borel space if it is homeomorphic to a Borel subset of a Polish space (i.e., a separable and completely metrizable space). Any Borel space $\mathcal{S}$ is assumed to be endowed with a Borel sigma-algebra, which is denoted by $\mathcal{B}(\mathcal{S})$. Examples of a Borel space are the Euclidean spaces $\mathbb{R}^{n}$, its Borel subsets endowed with a subspace topology, as well as hybrid spaces. We consider a map $f: \mathcal{S} \rightarrow Y$ measurable whenever it is Borel measurable.

We also assume that triple $\left(\Omega, \mathcal{F}_{\Omega}, \mathbb{P}_{\Omega}\right)$ denotes a probability space endowed with a filtration $\mathbb{F}=\left(\mathcal{F}_{s}\right)_{s \geq 0}$ satisfying the usual conditions of completeness and right continuity. Let $\left(\mathbb{W}_{s}\right)_{s \geq 0}$ be a b-dimensional $\mathbb{F}$-Brownian motion, and ${ }_{35}\left(\mathbb{P}_{s}\right)_{s \geq 0}$ be an $r$-dimensional $\mathbb{F}$-Poisson process. We assume that the Poisson process and Brownian motion are independent of each other. The Poisson process $\mathbb{P}_{s}=\left[\mathbb{P}_{s}^{1} ; \cdots ; \mathbb{P}_{s}^{r}\right]$ models $r$ events whose occurrences are assumed to be independent of each other.

\subsection{Notation}

We employ the following notation throughout the paper. We denote the set of nonnegative integers by $\mathbb{N}:=\{0,1,2, \ldots\}$ and the set of positive integers by $\mathbb{N}_{\geq 1}:=\{1,2,3, \ldots\}$. The symbols $\mathbb{R}, \mathbb{R}_{>0}$, and $\mathbb{R}_{\geq 0}$ denote the set of real, positive and nonnegative real numbers, respectively. We employ $x=\left[x_{1} ; \ldots ; x_{N}\right]$ to denote the corresponding vector of dimension $\sum_{i} n_{i}$, given 
for any $i \in\{1, \ldots, N\}$, their Cartesian product $\prod_{i=1}^{N} f_{i}: \prod_{i=1}^{N} X_{i} \rightarrow \prod_{i=1}^{N} Y_{i}$ is defined as $\left(\prod_{i=1}^{N} f_{i}\right)\left(x_{1}, \ldots, x_{N}\right)=\left[f_{1}\left(x_{1}\right) ; \ldots ; f_{N}\left(x_{N}\right)\right]$. We denote by $\|\cdot\|$ and $\|\cdot\|_{2}$ the infinity and Euclidean norms, respectively. Given a measurable function $f: \mathbb{N} \rightarrow \mathbb{R}^{n}$, the (essential) supremum of $f$ is denoted by $\|f\|_{\infty}:=$ (ess) $\sup \{\|f(k)\|, k \geq 0\}$. The identity matrix in $\mathbb{R}^{n \times n}$ is denoted by $\mathbb{I}_{n}$. The column vectors in $\mathbb{R}^{n \times 1}$ with all elements equal to zero and one are denoted by $\mathbf{0}_{n}$ and $\mathbb{1}_{n}$, respectively. The identity function and composition of functions are respectively denoted by $\mathcal{I}_{d}$ and symbol o. A function $\gamma: \mathbb{R}_{\geq 0} \rightarrow \mathbb{R}_{\geq 0}$, is said to be a class $\mathcal{K}$ function if it is continuous, strictly increasing, and $\gamma(0)=0$. A

class $\mathcal{K}$ function $\gamma(\cdot)$ is said to be a class $\mathcal{K}_{\infty}$ if $\gamma(r) \rightarrow \infty$ as $r \rightarrow \infty$.

\subsection{Continuous-Time Stochastic Hybrid Systems}

We consider stochastic hybrid systems in continuous-time (ct-SHS) defined over a general state space as in the following definition.

Definition 2.1. A continuous-time stochastic hybrid system (ct-SHS) in this work is characterized by the tuple

$$
\Sigma=(X, U, W, \mathcal{U}, \mathcal{W}, f, \sigma, \rho, Y, h)
$$

where:

- $X \subseteq \mathbb{R}^{n}$ is the state space of the system;

- $U \subseteq \mathbb{R}^{m}$ is the external input space of the system;

- $W \subseteq \mathbb{R}^{p}$ is the internal input space of the system;

- $\mathcal{U}$ and $\mathcal{W}$ are subsets of the sets of all $\mathbb{F}$-progressively measurable processes taking values in $\mathbb{R}^{m}$ and $\mathbb{R}^{p}$;

- $f: X \times U \times W \rightarrow X$ is the drift term which is globally Lipschitz continuous: there exist constants $\mathscr{L}_{x}, \mathscr{L}_{\nu}, \mathscr{L}_{w} \in \mathbb{R}_{\geq 0}$ such that $\| f(x, \nu, w)-$ $f\left(x^{\prime}, \nu^{\prime}, w^{\prime}\right)\left\|\leq \mathscr{L}_{x}\right\| x-x^{\prime}\left\|+\mathscr{L}_{\nu}\right\| \nu-\nu^{\prime}\left\|+\mathscr{L}_{w}\right\| w-w^{\prime} \|$ for all $x, x^{\prime} \in X$, for all $\nu, \nu^{\prime} \in U$, and for all $w, w^{\prime} \in W$; 
- $\sigma: \mathbb{R}^{n} \rightarrow \mathbb{R}^{n \times b}$ is the diffusion term which is globally Lipschitz continuous with the Lipschitz constant $\mathscr{L}_{\sigma}$;

- $\rho: \mathbb{R}^{n} \rightarrow \mathbb{R}^{n \times r}$ is the reset term which is globally Lipschitz continuous with the Lipschitz constant $\mathscr{L}_{\rho}$;

- $Y \subseteq \mathbb{R}^{q}$ is the output space of the system;

- $h: X \rightarrow Y$ is the output map.

A continuous-time stochastic hybrid system $\Sigma$ satisfies

$$
\Sigma:\left\{\begin{array}{l}
\mathrm{d} \xi(t)=f(\xi(t), \nu(t), w(t)) \mathrm{d} t+\sigma(\xi(t)) \mathrm{d} \mathbb{W}_{t}+\rho(\xi(t)) \mathrm{d} \mathbb{P}_{t}, \\
\zeta(t)=h(\xi(t))
\end{array}\right.
$$

$\mathbb{P}$-almost surely (P-a.s.) for any $\nu \in \mathcal{U}$ and any $w \in \mathcal{W}$, where stochastic processes $\xi: \Omega \times \mathbb{R}_{\geq 0} \rightarrow X$ and $\zeta: \Omega \times \mathbb{R}_{\geq 0} \rightarrow Y$ are called the solution process and the output trajectory of $\Sigma$, respectively. We also employ $\xi_{a \nu w}(t)$ to denote the value of the solution process at time $t \in \mathbb{R}_{\geq 0}$ under input trajectories $\nu$ and $w$ from initial condition $\xi_{a \nu w}(0)=a \mathbb{P}$-a.s., where $a$ is a random variable that ${ }_{180}$ is $\mathcal{F}_{0}$-measurable. We also denote by $\zeta_{a \nu w}$ the output trajectory corresponding to solution process $\xi_{a \nu w}$. Here, we assume that the Poisson processes $\mathbb{P}_{s}^{z}$, for any $z \in[1 ; \mathrm{r}]$, have the rates $\lambda_{z}$. We emphasize that the postulated assumptions on $f, \sigma$, and $\rho$ ensure existence, uniqueness, and strong Markov property of the solution process 32 .

185 Remark 2.2. Note that the underlying dynamic considered in (2) is a class of stochastic hybrid systems in which the drift and diffusion terms model the continuous part and the Poisson process models the discrete jump of the system. In fact, as showed in [7], linear stochastic hybrid automata are a class of the systems defined in (2). The employed notion of hybrid systems here is different from the one presented in [33].

Remark 2.3. In this paper, we are ultimately interested in investigating continuoustime stochastic hybrid systems with possibly large-state dimensions but without 


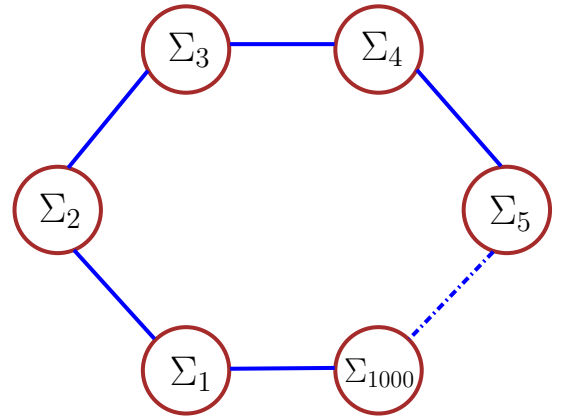

Figure 1: A circular building in a network of 1000 rooms.

internal inputs. In this case, the tuple (1) reduces to $(X, U, \mathcal{U}, f, \sigma, \rho, Y, h)$ with $f: X \times U \rightarrow X$, and ct-SHS (2) can be re-written as

$$
\Sigma:\left\{\begin{array}{l}
\mathrm{d} \xi(t)=f(\xi(t), \nu(t)) \mathrm{d} t+\sigma(\xi(t)) \mathrm{d} \mathbb{W}_{t}+\rho(\xi(t)) \mathrm{d} \mathbb{P}_{t}, \\
\zeta(t)=h(\xi(t)) .
\end{array}\right.
$$

The interconnected stochastic hybrid systems, defined later in Subsection 4.1. are also a class of hybrid systems without internal inputs, resulting from the interconnection of ct-SHSs with both internal and external inputs.

For the sake of better illustration of the results, we provide our case study as a running example throughout the paper.

Case Study. Consider a network of $n=1000$ rooms each equipped with a heater and connected circularly as depicted in Figure 1. The model of this case study is adapted from 34 by including nonlinearity and stochasticity in the model. The evolution of the temperature $T(\cdot)$ can be described by the interconnected stochastic differential equation

$$
\Sigma:\left\{\begin{array}{l}
\mathrm{d} T(t)=\left(A T(t)+\theta T_{h} \nu(t)+\bar{\beta} T_{E}+\varphi(\xi(t)) \mathrm{d} t+G \mathrm{~d} \mathbb{W}_{t}+R \mathrm{~d} \mathbb{P}_{t},\right. \\
\zeta(t)=T(t),
\end{array}\right.
$$

where $A$ is a matrix with diagonal elements $a_{i i}=-2 \eta-\bar{\beta}, i \in\{1, \ldots, n\}$, off-diagonal elements $a_{i, i+1}=a_{i+1, i}=a_{1, n}=a_{n, 1}=\eta, i \in\{1, \ldots, n-1\}$, and all other elements are identically zero, $G=0.5 \mathbb{I}_{n}, R=0.2 \mathbb{I}_{n}$, and $\varphi(\xi(t))=$ $\left[0.5 \varphi_{1}\left(0.5 \xi_{1}(t)\right) ; \ldots ; 0.5 \varphi_{n}\left(0.5 \xi_{n}(t)\right)\right]$ with $\varphi_{i}(x)=\sin (x), \forall i \in\{1, \ldots, n\}$. Pa200 rameters $\eta=0.05, \bar{\beta}=0.005$, and $\theta=0.01$ are conduction factors, respectively, 
between the rooms $i \pm 1$ and $i$, the external environment and the room $i$, and the heater and the room $i$. Moreover, $T_{E}=\left[T_{e_{1}} ; \ldots ; T_{e_{n}}\right], \nu(t)=\left[\nu_{1}(t) ; \ldots ; \nu_{n}(t)\right]$, and $T(t)=\left[T_{1}(t) ; \ldots ; T_{n}(t)\right]$, where $T_{i}(t)$ is taking values in the set $[20,21]$, for all $i \in\{1, \ldots, n\}$. Outside temperatures are the same for all rooms: $T_{e i}=$ ${ }_{205}-1{ }^{\circ} \mathrm{C}, \forall i \in\{1, \ldots, n\}$, and the heater temperature is $T_{h}=50^{\circ} \mathrm{C}$.

\subsection{Discrete-Time Finite Abstractions of ct-SHS}

In this subsection, we discuss discrete-time finite abstractions (also known as finite Markov decision processes) of continuous-time stochastic hybrid systems. Note that in the setting of this paper, the infinite abstraction refers to an approximation of the original system in the discrete-time but continuousspace domain, while the finite abstraction is another approximation in both discrete-time and discrete state set. In order to propose the construction procedure for finite abstractions, we first need to introduce infinite abstractions as time-discretized versions of ct-SHS in (2) as defined next, because our finite abstractions are constructed from the infinite discrete-time counterparts.

Definition 2.4. A discrete-time infinite abstraction of ct-SHS $\Sigma$ is denoted by the tuple

$$
\widetilde{\Sigma}=(\tilde{X}, \tilde{U}, \tilde{W}, \varsigma, \tilde{f}, \tilde{Y}, \tilde{h}),
$$

where:

- $\tilde{X} \subseteq \mathbb{R}^{n}$ is a Borel space as the state space of the system. We denote by $(\tilde{X}, \mathcal{B}(\tilde{X}))$ the measurable space with $\mathcal{B}(\tilde{X})$ being the Borel sigma-algebra on the state space;

220 $\quad \tilde{U} \subseteq \mathbb{R}^{m}$ is a Borel space as the external input space of the system;

- $\tilde{W} \subseteq \mathbb{R}^{p}$ is a Borel space as the internal input space of the system;

- $\varsigma$ is a sequence of independent and identically distributed (i.i.d.) random variables from a sample space $\Omega$ to the set $V_{\varsigma}$,

$$
\varsigma:=\left\{\varsigma(k): \Omega \rightarrow V_{\varsigma}, k \in \mathbb{N}\right\}
$$


- $\tilde{f}: \tilde{X} \times \tilde{U} \times \tilde{W} \times V_{\varsigma} \rightarrow \tilde{X}$ is a measurable function characterizing the state evolution of the system;

- $\tilde{Y} \subseteq \mathbb{R}^{q}$ is a Borel space as the output space of the system;

- $\tilde{h}: \tilde{X} \rightarrow \tilde{Y}$ is a measurable function that maps a state $\tilde{x} \in \tilde{X}$ to its output.

For given initial state $\tilde{x}(0) \in \tilde{X}$ and input sequences $\{\tilde{\nu}(k): \Omega \rightarrow \tilde{U}, k \in \mathbb{N}\}$ and $\{\tilde{w}(k): \Omega \rightarrow \tilde{W}, k \in \mathbb{N}\}$, evolution of $\widetilde{\Sigma}$ can be written as

$$
\widetilde{\Sigma}:\left\{\begin{array}{l}
\tilde{\xi}(k+1)=\tilde{f}(\tilde{\xi}(k), \tilde{\nu}(k), \tilde{w}(k), \varsigma(k)), \quad k \in \mathbb{N} . \\
\tilde{\zeta}(k)=\tilde{h}(\tilde{\xi}(k)),
\end{array}\right.
$$

We associate to $\tilde{U}$ and $\tilde{W}$ the sets $\tilde{\mathcal{U}}$ and $\tilde{\mathcal{W}}$ to be the collections of sequences $\{\tilde{\nu}(k): \Omega \rightarrow \tilde{U}, k \in \mathbb{N}\}$ and $\{\tilde{w}(k): \Omega \rightarrow \tilde{W}, k \in \mathbb{N}\}$, in which $\tilde{\nu}(k)$ and $\tilde{w}(k)$ are independent of $\varsigma(t)$ for any $k, t \in \mathbb{N}$ and $t \geq k$. For any initial state $\tilde{a} \in \tilde{X}, \tilde{\nu}(\cdot) \in \tilde{\mathcal{U}}$ and $\tilde{w}(\cdot) \in \tilde{\mathcal{W}}$, the random sequences $\tilde{\xi}_{\tilde{a} \tilde{\nu} \tilde{w}}: \Omega \times \mathbb{N} \rightarrow \tilde{X}$, $\tilde{\zeta}_{\tilde{a} \tilde{\nu} \tilde{w}}: \Omega \times \mathbb{N} \rightarrow \tilde{Y}$ satisfying (5) are respectively called the solution process and output trajectory of $\widetilde{\Sigma}$ under external input $\tilde{\nu}$, internal input $\tilde{w}$, and initial state $\tilde{a}$.

A discrete-time infinite abstraction of ct-SHS $\Sigma$ in (4) can be equivalently represented as a Markov decision process (MDP) 35.

$$
\widetilde{\Sigma}=\left(\tilde{X}, \tilde{U}, \tilde{W}, \tilde{T}_{\tilde{x}}, \tilde{Y}, \tilde{h}\right)
$$

where the map $\tilde{T}_{\tilde{x}}: \mathcal{B}(\tilde{X}) \times \tilde{X} \times \tilde{U} \times \tilde{W} \rightarrow[0,1]$, is a conditional stochastic kernel that assigns to any $\tilde{x} \in \tilde{X}, \tilde{\nu} \in \tilde{U}$, and $\tilde{w} \in \tilde{W}$, a probability measure $\tilde{T}_{\tilde{x}}(\cdot \mid \tilde{x}, \tilde{\nu}, \tilde{w})$ on the measurable space $(\tilde{X}, \mathcal{B}(\tilde{X}))$ so that for any set $\mathcal{A} \in \mathcal{B}(\tilde{X})$,

$$
\mathbb{P}(\tilde{x}(k+1) \in \mathcal{A} \mid \tilde{x}(k), \tilde{\nu}(k), \tilde{w}(k))=\int_{\mathcal{A}} \tilde{T}_{\tilde{x}}(\mathrm{~d} \tilde{x}(k+1) \mid \tilde{x}(k), \tilde{\nu}(k), \tilde{w}(k)) .
$$

For given inputs $\tilde{\nu}(\cdot), \tilde{w}(\cdot)$, the stochastic kernel $\tilde{T}_{\tilde{x}}$ captures the evolution of the state of $\widetilde{\Sigma}$ and can be uniquely determined by the pair $(\varsigma, \tilde{f})$ from (4).

Given the discrete-time stochastic hybrid system presented in (5), we are interested in the following Markov policies to control the system. 
Definition 2.5 ([36]). A Markov policy for the discrete-time stochastic hybrid system $\widetilde{\Sigma}$ in (5) is a sequence $\bar{\mu}=\left(\bar{\mu}_{0}, \bar{\mu}_{1}, \bar{\mu}_{2}, \ldots\right)$ of universally measurable stochastic kernels $\bar{\mu}_{n}$, each defined on the input space $\tilde{U}$ given $\tilde{X} \times \tilde{W}$. The class of all such Markov policies is denoted by $\mathcal{M}_{p}$.

A Markov policy is very similar to the notion of state feedback in control theory. According to the above definition, the Markov policy observes the exact values of state $\tilde{\xi}(n) \in \tilde{X}$ and internal input $\tilde{w}(n) \in \tilde{W}$ at time step $n$, and selects the external input $\tilde{\nu}(n) \in \tilde{U}$ as a sample from the probability measure ${ }_{245} \bar{\mu}_{n}(\cdot \mid \tilde{\xi}(n), \tilde{w}(n))$.

Now we proceed with constructing finite MDPs $\widehat{\Sigma}$ as finite abstractions of the discrete-time stochastic hybrid systems $\widetilde{\Sigma}$ presented in (5). The abstraction algorithm is adapted from [37 with some modifications. We assume the state and input sets of $\widetilde{\Sigma}$ are restricted to compact subsets over which we are interested to perform the synthesis. The rest of the state sets can be considered as single absorbing states in both $\widetilde{\Sigma}$ and $\widehat{\Sigma}$. In order to make the notation easier, we assume this procedure is already applied to the system and eliminate the absorbing states from the presentation. Then the abstraction algorithm in this work is based on finite partitions of sets $\tilde{X}=\cup_{z} \mathrm{X}_{z}, \tilde{U}=\cup_{z} \cup_{z}$, and $\tilde{W}=\cup_{z} \mathrm{~W}_{z}$ and selection of representative points $\bar{\xi}_{z} \in \mathrm{X}_{z}, \bar{\nu}_{z} \in \mathrm{U}_{z}$, and $\bar{w}_{z} \in \mathrm{W}_{z}$ as abstract states and inputs as in the following definition.

Definition 2.6. Given a ct-SHS $\Sigma=(X, U, W, \mathcal{U}, \mathcal{W}, f, \sigma, \rho, Y, h)$ with its timediscretized version $\widetilde{\Sigma}=(\tilde{X}, \tilde{U}, \tilde{W}, \varsigma, \tilde{f}, \tilde{Y}, \tilde{h})$, the finite abstraction $\widehat{\Sigma}$ can be represented as

$$
\widehat{\Sigma}=(\hat{X}, \hat{U}, \hat{W}, \varsigma, \hat{f}, \hat{Y}, \hat{h})
$$

where $\hat{X}=\left\{\bar{\xi}_{z}, z=1, \ldots, n_{\tilde{\xi}}\right\}, \hat{U}=\left\{\bar{\nu}_{z}, z=1, \ldots, n_{\tilde{\nu}}\right\}$, and $\hat{W}=\left\{\bar{w}_{z}, z=\right.$ $\left.1, \ldots, n_{\tilde{w}}\right\}$ are the sets of selected representative points. Function $\hat{f}: \hat{X} \times \hat{U} \times$ $\hat{W} \times V_{\varsigma} \rightarrow \hat{X}$ is defined as

$$
\hat{f}(\hat{\xi}, \hat{\nu}, \hat{w}, \varsigma)=\Phi_{\tilde{\xi}}(\tilde{f}(\hat{\xi}, \hat{\nu}, \hat{w}, \varsigma))
$$

where $\Phi_{\tilde{\xi}}: \tilde{X} \rightarrow \hat{X}$ is the map that assigns to any $\tilde{\xi} \in \tilde{X}$, the representative 
point $\bar{\xi} \in \hat{X}$ of the corresponding partition set containing $\tilde{\xi}$. The output map $\hat{h}$ is the same as $\tilde{h}$ with its domain restricted to finite state set $\hat{X}$ and the output set $\hat{Y}$ is just the image of $\hat{X}$ under $\tilde{h}$. The initial state of $\widehat{\Sigma}$ is also selected according to $\hat{\xi}_{0}:=\Phi_{\tilde{\xi}}\left(\tilde{\xi}_{0}\right)$ with $\tilde{\xi}_{0}$ being the initial state of $\widetilde{\Sigma}$.

Abstraction map $\Phi_{\tilde{\xi}}$ presented in (7) satisfies the inequality

$$
\left\|\Phi_{\tilde{\xi}}(\tilde{\xi})-\tilde{\xi}\right\| \leq \delta, \quad \forall \tilde{\xi} \in \tilde{X},
$$

where $\delta$ is the state discretization parameter defined as $\delta:=\sup \left\{\left\|\tilde{\xi}-\tilde{\xi}^{\prime}\right\|, \tilde{\xi}, \tilde{\xi}^{\prime} \in\right.$ $\left.\mathrm{X}_{z}, z=1,2, \ldots, n_{\tilde{\xi}}\right\}$.

Remark 2.7. Note that there is no restriction on discretizing the state, external and internal input sets. However, the size of the state discretization parameter $\delta$ appears in the formulated error ( $c f .35)$ ): one can decrease the error by reducing the state discretization parameter. We also do not have any constraint on the shape of the partition elements in constructing the finite MDPs. For the sake of easy implementation, one can consider the partition sets as boxes and the center of each box as representative points.

Remark 2.8. Note that in order to satisfy the abstraction condition proposed in (8) with bounded $\delta$, we need to restrict ourselves to compact subsets of the state and input spaces, and construct finite partitions from them.

A schematic relation between $\Sigma, \widetilde{\Sigma}$, and $\widehat{\Sigma}$ is depicted in Figure 2 In the next sections, we provide a framework for compositional synthesis of interconnected discrete-time (finite or infinite) abstractions from ct-SHS. We define notions of stochastic pseudo-simulation and simulation functions for quantifying the probabilistic error between the original continuous-time stochastic hybrid systems and that of their discrete-time (finite or infinite) abstractions with and without internal inputs, respectively.

\section{Stochastic Pseudo-Simulation and Simulation Functions}

In this section, we first introduce a notion of stochastic pseudo-simulation functions (SPSF) for ct-SHS with both internal and external inputs. We then 


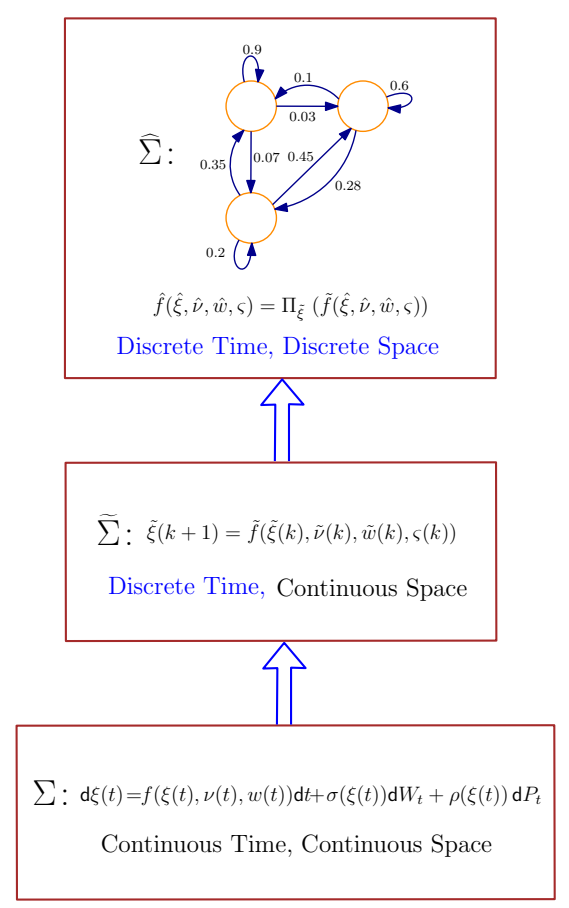

Figure 2: A schematic relation between $\Sigma, \widetilde{\Sigma}$, and $\widehat{\Sigma}$.

define a notion of stochastic simulation functions (SSF) for ct-SHS with only external inputs. We mainly employ these two definitions to quantify the probabilistic closeness of interconnected continuous-time stochastic hybrid systems and their discrete-time (finite or infinite) abstractions.

Definition 3.1. Consider a ct-SHS $\Sigma=(X, U, W, \mathcal{U}, \mathcal{W}, f, \sigma, \rho, Y, h)$ and its (in)finite abstraction $\widehat{\Sigma}=(\hat{X}, \hat{U}, \hat{W}, \varsigma, \hat{f}, \hat{Y}, \hat{h})$ with internal inputs. A function

${ }_{290} S: X \times \hat{X} \rightarrow \mathbb{R}_{\geq 0}$ is called a stochastic pseudo-simulation function (SPSF) from $\widehat{\Sigma}$ to $\Sigma$ if

- $\exists \alpha \in \mathcal{K}_{\infty}$ such that

$$
\forall x \in X, \forall \hat{x} \in \hat{X}, \quad \alpha(\|h(x)-\hat{h}(\hat{x})\|) \leq S(x, \hat{x}),
$$

- $\forall k \in \mathbb{N}, \forall \xi:=\xi(k \tau) \in X, \forall \hat{\xi}:=\hat{\xi}(k) \in \hat{X}$, and $\forall \hat{\nu}:=\hat{\nu}(k) \in \hat{U}, \forall w:=$ 


$$
\begin{aligned}
& w(k \tau) \in W, \forall \hat{w}:=\hat{w}(k) \in \hat{W}, \exists \nu:=\nu(k \tau) \in U \text { such that } \\
& \mathbb{E}[S(\xi((k+1) \tau), \hat{\xi}(k+1)) \mid \xi, \hat{\xi}, \nu, \hat{\nu}, w, \hat{w}] \\
& \leq \max \left\{\kappa S(\xi, \hat{\xi}), \rho_{\text {int }}(\|w-\hat{w}\|), \rho_{\text {ext }}(\|\hat{\nu}\|), \psi\right\},
\end{aligned}
$$

for some chosen sampling time $\tau \in \mathbb{R}_{>0}, 0<\kappa<1, \rho_{\text {ext }}, \rho_{\text {int }} \in \mathcal{K}_{\infty}$, and $\psi \in \mathbb{R}_{>0}$.

We write $\widehat{\Sigma} \preceq_{\mathcal{P S}} \Sigma$ if there exists an SPSF $S$ from $\widehat{\Sigma}$ to $\Sigma$, and call the hybrid 295 system $\widehat{\Sigma}$ a discrete-time (in)finite abstraction of concrete (original) system $\Sigma$. Abstraction $\widehat{\Sigma}$ could be finite or infinite depending on cardinalities of sets $\hat{X}, \hat{U}, \hat{W}$.

Remark 3.2. Note that since the concrete system in this work is considered in continuous-time domain, one can employ Dynkin's formula [38] and establish the following equality:

$$
\begin{aligned}
\mathbb{E} & {[S(\xi((k+1) \tau), \hat{\xi}(k+1)) \mid \xi(k \tau), \hat{\xi}(k), \nu(k \tau), \hat{\nu}(k), w(k \tau), \hat{w}(k)] } \\
& =\mathbb{E}_{\varsigma}\left[S(\xi(k \tau), \hat{\xi}(k+1))+\mathbb{E}\left[\int_{k \tau}^{(k+1) \tau} \mathcal{L} S(\xi(t), \hat{\xi}(k+1)) \mathrm{d} t\right] \mid \hat{\xi}(k), \hat{\nu}(k), \hat{w}(k)\right],
\end{aligned}
$$

where $\mathcal{L} S$ is the infinitesimal generator of the stochastic process acting on function $S$ [4], and $\mathbb{E}_{\varsigma}$ is the conditional expectation acting only on the noise of the abstract system. The above Dynkin's formula is employed later in Subsection 5.1 to show the results of Theorem 5.1.

Remark 3.3. Note that the above definition does not put any restriction on the state set of abstract systems; therefore, it can also be employed to establish a stochastic pseudo-simulation function from infinite abstractions $\widetilde{\Sigma}$ presented in (4) to $\Sigma$ (cf. the case study).

Remark 3.4. Condition 10 implicitly implies existence of a function $\nu(t)=$ $\nu_{\hat{\nu}}(\xi(k \tau), \hat{\xi}(k), w(k \tau), \hat{w}(k))$, for any $k \tau \leq t \leq(k+1) \tau$, fulfilling inequality 10). This function is called an interface function and can be employed to refine a synthesized policy $\hat{\nu}$ for $\widehat{\Sigma}$ to a policy $\nu$ for $\Sigma$. 
Now, we adapt the above notion to the interconnected ct-SHS without internal inputs by omitting all the terms related to $w, \hat{w}$ which will be utilized in Theorem 3.6 for relating interconnected systems.

Definition 3.5. Consider a ct-SHS $\Sigma=(X, U, \mathcal{U}, f, \sigma, \rho, Y, h)$ and its finite abstraction $\widehat{\Sigma}=(\hat{X}, \hat{U}, \varsigma, \hat{f}, \hat{Y}, \hat{h})$ without internal inputs. A function $V: X \times$ ${ }_{315} \hat{X} \rightarrow \mathbb{R}_{\geq 0}$ is called a stochastic simulation function (SSF) from $\widehat{\Sigma}$ to $\Sigma$ if

- $\exists \alpha \in \mathcal{K}_{\infty}$ such that

$$
\forall x \in X, \forall \hat{x} \in \hat{X}, \quad \alpha(\|h(x)-\hat{h}(\hat{x})\|) \leq V(x, \hat{x}),
$$

- $\forall k \in \mathbb{N}, \forall \xi:=\xi(k \tau) \in X, \forall \hat{\xi}:=\hat{\xi}(k) \in \hat{X}$, and $\forall \hat{\nu}:=\hat{\nu}(k) \in \hat{U}, \exists \nu:=$ $\nu(k \tau) \in U$ such that

$$
\mathbb{E}[V(\xi((k+1) \tau), \hat{\xi}(k+1)) \mid \xi, \hat{\xi}, \nu, \hat{\nu}] \leq \max \left\{\kappa V(\xi, \hat{\xi}), \rho_{\text {ext }}(\|\hat{\nu}\|), \psi\right\},
$$

for some chosen sampling time $\tau \in \mathbb{R}_{>0}, 0<\kappa<1, \rho_{\text {ext }} \in \mathcal{K}_{\infty}$, and $\psi \in \mathbb{R}_{>0}$.

We write $\widehat{\Sigma} \preceq \mathcal{S} \Sigma$ if there exists an SSF $V$ from $\widehat{\Sigma}$ to $\Sigma$, and call the hybrid system $\widehat{\Sigma}$ a discrete-time (in)finite abstraction of concrete (original) system $\Sigma$.

The next theorem shows how SSF can be utilized to compare output trajectories of original interconnected continuous-time stochastic hybrid systems and that of their discrete-time (finite or infinite) abstractions. This theorem is borrowed from [16, Theorem 3.3] with slight modification, and holds for the setting here since the max form of SSF here implies the additive form used in 325 [16].

Theorem 3.6. Let $\Sigma=(X, U, \mathcal{U}, f, \sigma, \rho, Y, h)$ be a ct-SHS and $\widehat{\Sigma}=(\hat{X}, \hat{U}, \varsigma, \hat{f}$, $\hat{Y}, \hat{h})$ its discrete-time abstraction without internal inputs. Suppose $V$ is an SSF from $\widehat{\Sigma}$ to $\Sigma$. For any input trajectory $\hat{\nu}(\cdot) \in \hat{\mathcal{U}}$ that preserves Markov property for the closed-loop $\widehat{\Sigma}$, and for any random variables a and $\hat{a}$ as the initial states of the ct-SHS and its discrete-time abstraction, there exists an input trajectory 
$\nu(\cdot) \in \mathcal{U}$ of $\Sigma$ through the interface function associated with $V$ such that the following inequality holds:

$$
\begin{aligned}
& \mathbb{P}\left\{\sup _{0 \leq k \leq T_{d}}\left\|\zeta_{a \nu}(k \tau)-\hat{\zeta}_{\hat{a} \hat{\nu}}(k)\right\| \geq \varepsilon \mid[a ; \hat{a}]\right\} \\
& \leq \begin{cases}1-\left(1-\frac{V(a, \hat{a})}{\alpha(\varepsilon)}\right)\left(1-\frac{\hat{\psi}}{\alpha(\varepsilon)}\right)^{T_{d}} & \text { if } \alpha(\varepsilon) \geq \frac{\hat{\psi}}{\kappa}, \\
\left(\frac{V(a, \hat{a})}{\alpha(\varepsilon)}\right)(1-\kappa)^{T_{d}}+\left(\frac{\hat{\psi}}{\kappa \alpha(\varepsilon)}\right)\left(1-(1-\kappa)^{T_{d}}\right) & \text { if } \alpha(\varepsilon)<\frac{\hat{\psi}}{\kappa},\end{cases}
\end{aligned}
$$

where constant $\hat{\psi}>0$ satisfies $\hat{\psi} \geq \rho_{\text {ext }}\left(\|\hat{\nu}\|_{\infty}\right)+\psi$.

\section{Compositional Abstractions for Interconnected Hybrid Systems}

In this section, we analyze networks of stochastic hybrid subsystems

$$
\Sigma_{i}=\left(X_{i}, U_{i}, W_{i}, \mathcal{U}_{i}, \mathcal{W}_{i}, f_{i}, \sigma_{i}, \rho_{i}, Y_{i}, h_{i}\right), \quad i \in\{1, \ldots, N\}
$$

and discuss how to construct their finite abstractions together with an SSF based on corresponding SPSF functions of their subsystems.

\subsection{Interconnected Stochastic Hybrid Systems}

We consider a collection of stochastic hybrid subsystems $\Sigma_{i}$ as in (14) where their internal inputs and outputs are partitioned as

$$
\begin{aligned}
w_{i} & =\left[w_{i 1} ; \ldots ; w_{i(i-1)} ; w_{i(i+1)} ; \ldots ; w_{i N}\right], \\
y_{i} & =\left[y_{i 1} ; \ldots ; y_{i N}\right]
\end{aligned}
$$

and their output spaces and functions are of the form

$$
Y_{i}=\prod_{j=1}^{N} Y_{i j}, \quad h_{i}\left(x_{i}\right)=\left[h_{i 1}\left(x_{i}\right) ; \ldots ; h_{i N}\left(x_{i}\right)\right] .
$$

The outputs $y_{i i}$ are interpreted as external ones, whereas the outputs $y_{i j}$ with $i \neq j$ are internal ones which are employed to interconnect these stochastic hybrid subsystems. For the interconnection, if there is a connection from $\Sigma_{j}$ to $\Sigma_{i}$, we assume that $w_{i j}$ is equal to $y_{j i}$. Otherwise, we put the connecting interconnected stochastic hybrid systems. 
Definition 4.1. Consider $N \in \mathbb{N}_{\geq 1}$ stochastic hybrid subsystems $\Sigma_{i}=\left(X_{i}, U_{i}\right.$ , $\left.W_{i}, \mathcal{U}_{i}, \mathcal{W}_{i}, f_{i}, \sigma_{i}, \rho_{i}, Y_{i}, h_{i}\right), i \in\{1, \ldots, N\}$, with the input-output configuration as in (15) and (16). The interconnection of $\Sigma_{i}$ for any $i \in\{1, \ldots, N\}$, is the concrete interconnected stochastic hybrid system $\Sigma=(X, U, \mathcal{U}, f, \sigma, \rho, Y, h)$, denoted by $\mathcal{I}\left(\Sigma_{1}, \ldots, \Sigma_{N}\right)$, such that $X:=\prod_{i=1}^{N} X_{i}, U:=\prod_{i=1}^{N} U_{i}, f:=\prod_{i=1}^{N} f_{i}$, $\sigma:=\left[\sigma_{1}\left(x_{1}\right) ; \cdots ; \sigma_{N}\left(x_{N}\right)\right], \rho:=\left[\rho_{1}\left(x_{1}\right) ; \cdots ; \rho_{N}\left(x_{N}\right)\right], Y:=\prod_{i=1}^{N} Y_{i i}$, and $h=\prod_{i=1}^{N} h_{i i}$, subject to the following constraint:

$$
\forall i, j \in\{1, \ldots, N\}, i \neq j: \quad w_{j i}=y_{i j}, \quad Y_{i j} \subseteq W_{j i} .
$$

Remark 4.2. Note that we employ the term "internal" for inputs and outputs of subsystems that are affecting each other in the interconnection: internal output of a subsystem affects internal input of another subsystem. We utilize the term "external" for inputs and outputs that are not used for the sake of constructing the interconnection. Properties of the interconnected system are specified over the external outputs. The main goal is to synthesize external inputs to satisfy desired properties over external outputs.

An example of the interconnection of two concrete stochastic hybrid subsystems $345 \quad \Sigma_{1}$ and $\Sigma_{2}$ is illustrated in Figure 3 .

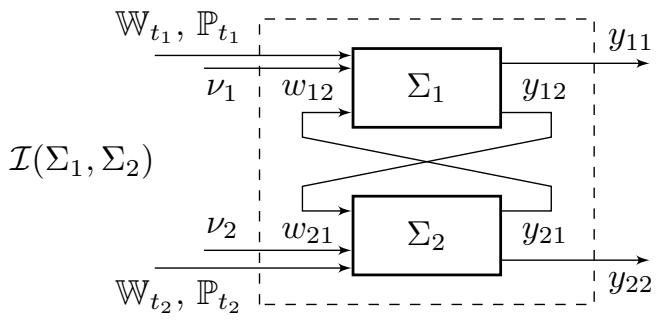

Figure 3: Interconnection of two concrete stochastic hybrid subsystems $\Sigma_{1}$ and $\Sigma_{2}$.

Case Study (Continued). By considering the individual rooms as 


\section{$\Sigma_{i}$ described by}

$\Sigma_{i}:\left\{\begin{aligned} & \mathrm{d} T_{i}(t)=\left(a_{i i} T_{i}(t)+\theta T_{h} \nu_{i}(t)+\eta w_{i}(t)+\bar{\beta} T_{e_{i}}+0.5 \varphi_{i}\left(0.5 \xi_{i}(t)\right)\right) \mathrm{d} t+0.5 \mathrm{~d} \mathbb{W}_{t_{i}} \\ &+0.2 \mathrm{dP}_{t_{i}} \\ & \zeta_{i}(t)=T_{i}(t)\end{aligned}\right.$

one can readily verify that $\Sigma=\mathcal{I}\left(\Sigma_{1}, \ldots, \Sigma_{N}\right)$ where $w_{i}(t)=\left[\zeta_{i-1}(t) ; \zeta_{i+1}(t)\right]$

(with $\left.\zeta_{0}=\zeta_{n}, \zeta_{n+1}=\zeta_{1}\right)$.

\subsection{Compositional Abstractions of Interconnected Hybrid Systems}

In this subsection, we consider $\Sigma_{i}=\left(X_{i}, U_{i}, W_{i}, \mathcal{U}_{i}, \mathcal{W}_{i}, f_{i}, \sigma_{i}, \rho_{i}, Y_{i}, h_{i}\right)$ as an original ct-SHS and $\widehat{\Sigma}$ as its discrete-time finite abstraction given by the tuple $\widehat{\Sigma}_{i}=\left(\hat{X}_{i}, \hat{U}_{i}, \hat{W}_{i}, \varsigma_{i}, \hat{f}_{i}, \hat{Y}_{i}, \hat{h}_{i}\right)$ with the input-output configuration similar to 15 and (16), where $\hat{W}_{i} \subseteq W_{i}$ and $\hat{Y}_{i} \subseteq Y_{i}$. In order to present the compositionality results of the paper, we assume there exist SPSF $S_{i}$ from $\widehat{\Sigma}_{i}$ to $\Sigma_{i}$ satisfying conditions (9), 10 in Definition 3.1 with the corresponding functions and constants denoted by $\alpha_{i}, \rho_{\text {int } i}, \rho_{\text {ext } i}, \kappa_{i}$, and $\psi_{i}$. Since we construct our finite MDPs $\widehat{\Sigma}_{i}$ from time-discretized versions of original systems (i.e. from $\widetilde{\Sigma}_{i}$ ), we define here the abstraction map $\Phi_{\tilde{w}_{j i}}$ on $\tilde{W}_{j i}$ that assigns to any $\tilde{w}_{j i} \in \tilde{W}_{j i}$ representative point $\bar{w}_{j i} \in \hat{W}_{j i}$ of the corresponding partition set containing $\tilde{w}_{j i}$. The mentioned map satisfies

$$
\left\|\Phi_{\tilde{w}_{j i}}\left(\tilde{w}_{j i}\right)-\tilde{w}_{j i}\right\| \leq \mu_{j i}, \quad \forall \tilde{w}_{j i} \in \tilde{W}_{j i}
$$

where $\mu_{j i}$ is an internal input discretization parameter defined similar to $\delta$ in (8). Now we define a notion of interconnection applicable to discrete-time finite abstractions. Note that condition (17) helps us to freely take quantization parameters of internal input sets at the cost of having an additional error term formulated in $\psi$ in 31 .

Definition 4.3. Consider $N \in \mathbb{N}_{\geq 1}$ finite stochastic hybrid subsystems $\widehat{\Sigma}_{i}=$ $\left(\hat{X}_{i}, \hat{U}_{i}, \hat{W}_{i}, \varsigma_{i}, \hat{f}_{i}, \hat{Y}_{i}, \hat{h}_{i}\right), i \in\{1, \ldots, N\}$. The interconnection of $\widehat{\Sigma}_{i}$ is the finite interconnected stochastic hybrid system $\widehat{\Sigma}=(\hat{X}, \hat{U}, \varsigma, \hat{f}, \hat{Y}, \hat{h})$, denoted by $\widehat{\mathcal{I}}\left(\widehat{\Sigma}_{1}, \ldots, \widehat{\Sigma}_{N}\right)$, such that $\hat{X}:=\prod_{i=1}^{N} \hat{X}_{i}, \hat{U}:=\prod_{i=1}^{N} \hat{U}_{i}, \varsigma:=\left[\varsigma_{1}, \ldots, \varsigma_{N}\right]$, 
$\hat{f}:=\prod_{i=1}^{N} \hat{f}_{i}, \hat{Y}:=\prod_{i=1}^{N} \hat{Y}_{i i}$, and $\hat{h}=\prod_{i=1}^{N} \hat{h}_{i i}$, subject to the following constraint:

$$
\forall i, j \in\{1, \ldots, N\}, i \neq j: \hat{w}_{j i}=\Phi_{\tilde{w}_{j i}}\left(\hat{y}_{i j}\right), \quad \Phi_{\tilde{w}_{j i}}\left(\hat{Y}_{i j}\right) \subseteq \hat{W}_{j i}
$$

Now we raise the following small-gain assumption that is essential for the compositionality result in this section.

Assumption 1. Assume that there exist $\mathcal{K}_{\infty}$ functions $\tilde{\delta}_{f}, \bar{\lambda}$ such that $(\bar{\lambda}-$ $\left.\mathcal{I}_{d}\right) \in \mathcal{K}_{\infty}$ and $\mathcal{K}_{\infty}$ functions $\kappa_{i j}$ defined as

$$
\kappa_{i j}(s):= \begin{cases}\kappa_{i} s & \text { if } i=j, \\ \left(\mathcal{I}_{d}+\tilde{\delta}_{f}\right) \circ \rho_{\mathrm{int} i} \circ \bar{\lambda} \circ \alpha_{j}^{-1}(s) & \text { if } i \neq j,\end{cases}
$$

satisfy

$$
\kappa_{i_{1} i_{2}} \circ \kappa_{i_{2} i_{3}} \circ \cdots \circ \kappa_{i_{r-1} i_{r}} \circ \kappa_{i_{r} i_{1}}<\mathcal{I}_{d}
$$

for all sequences $\left(i_{1}, \ldots, i_{r}\right) \in\{1, \ldots, N\}^{r}$ and $r \in\{1, \ldots, N\}$.

The small-gain condition (18) implies the existence of $\mathcal{K}_{\infty}$ functions $\bar{\sigma}_{i}$ [39, Theorem 5.5], satisfying

$$
\max _{i, j}\left\{\bar{\sigma}_{i}^{-1} \circ \kappa_{i j} \circ \bar{\sigma}_{j}\right\}<\mathcal{I}_{d}, \quad i, j=\{1, \ldots, N\} .
$$

Remark 4.4. Note that since $\alpha_{j}, \sigma_{i}$ in Assumption 1 are $\mathcal{K}_{\infty}$ functions and consequently strictly increasing, their inverses always exist.

Remark 4.5. Note that the small-gain condition (18) is a standard one in studying the stability of large-scale interconnected systems via input-to-state stable Lyapunov functions [40, 41].

This condition is automatically satisfied if each $\kappa_{i i}$ is less than identity $\left(\kappa_{i i}<\right.$ $\left.\mathcal{I}_{d}, \forall i \in\{1, \ldots, N\}\right)$. Since this condition should be satisfied for all possible sequences $\left(i_{1}, \ldots, i_{r}\right) \in\{1, \ldots, N\}^{r}, r \in\{1, \ldots, N\}$, it allows some subsystems to compensate the undesirable effects of other subsystems in the interconnected network such that this condition is satisfied. 
In the next theorem, we employ small-gain Assumption 1 to quantify the error between the interconnection of continuous-time stochastic hybrid subsystems and that of their discrete-time abstractions in a compositional manner.

Theorem 4.6. Consider an interconnected ct-SHS $\Sigma=\mathcal{I}\left(\Sigma_{1}, \ldots, \Sigma_{N}\right)$ induced by $N \in \mathbb{N}_{\geq 1}$ continuous-time stochastic hybrid subsystems $\Sigma_{i}$. Suppose that each $\Sigma_{i}$ admits a discrete-time abstraction $\widehat{\Sigma}_{i}$ together with an SPSF $S_{i}$. If Assumption 1 holds and $\max _{i} \bar{\sigma}_{i}^{-1}$ for $\bar{\sigma}_{i}$ as in 19 is concave, then function $V(x, \hat{x})$ defined as

$$
V(x, \hat{x}):=\max _{i}\left\{\bar{\sigma}_{i}^{-1}\left(S_{i}\left(x_{i}, \hat{x}_{i}\right)\right)\right\}
$$

370 is an $S S F$ from $\widehat{\Sigma}=\widehat{\mathcal{I}}\left(\widehat{\Sigma}_{1}, \ldots, \widehat{\Sigma}_{N}\right)$ to $\Sigma=\mathcal{I}\left(\Sigma_{1}, \ldots, \Sigma_{N}\right)$.

The proof of Theorem 4.6 is provided in the Appendix.

The results of Theorem 4.6 are schematically depicted in Figure 4 As illustrated, if there exists an SPSF $S_{i}\left(x_{i}, \hat{x}_{i}\right)$ between each original subsystem and its corresponding finite MDP, one can construct an SSF $V(x, \hat{x})$ as proposed in 20 between the interconnected original system and its interconnected finite abstraction provided that the small-gain condition 18 is satisfied.

\section{Construction of Stochastic Pseudo-Simulation Functions}

In this section, we impose conditions on the infinite ct-SHS $\Sigma$ enabling us to

establish an SPSF from its finite abstraction $\widehat{\Sigma}$ to $\Sigma$. The required conditions are presented for a particular class of continuous-time nonlinear stochastic hybrid systems as in the next subsection. This is one of the contributions of our work compared to [31] in which only the class of linear affine systems is handled.

\subsection{A Class of Nonlinear Stochastic Hybrid Systems}

In this subsection, we focus on a special class of continuous-time nonlinear stochastic hybrid systems $\Sigma$ and quadratic pseudo-stochastic simulation functions $S$. First, we formally define this class of systems. Afterwards, we construct 


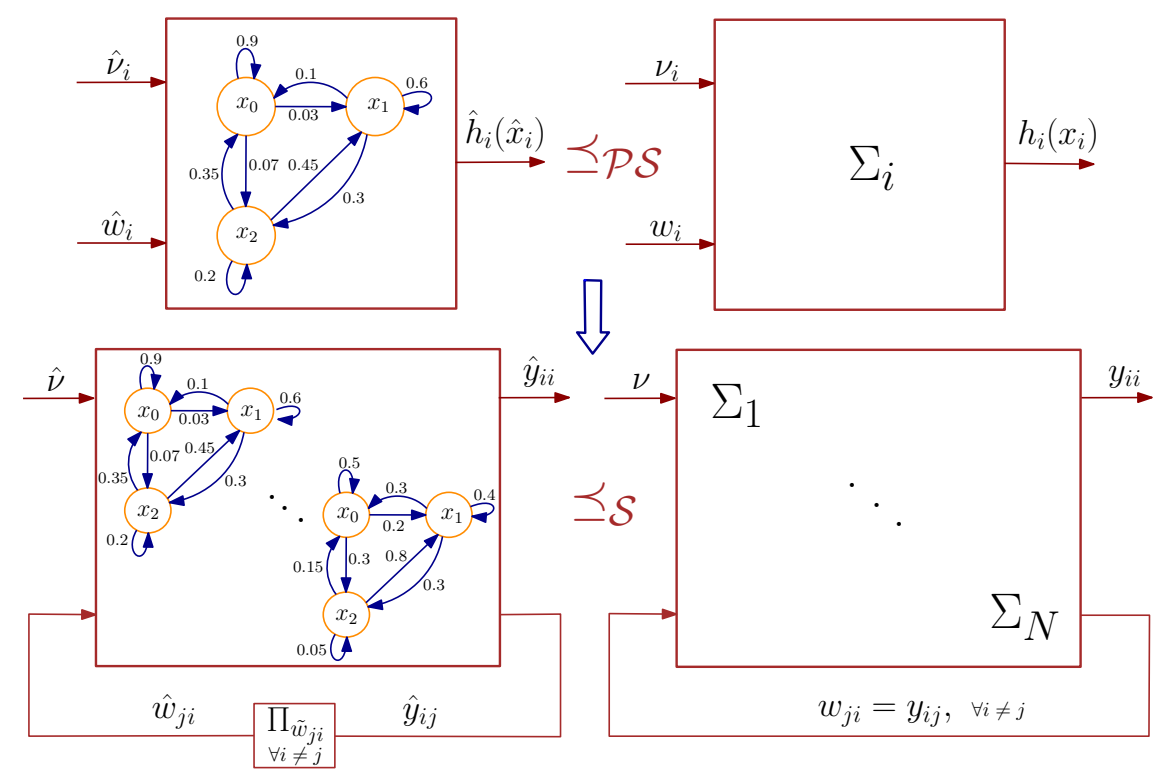

Figure 4: Compositionality result of the paper given that the small-gain condition 18 is satisfied.

their finite abstractions $\widehat{\Sigma}$ as discussed in Subsection 2.4 and then provide conditions under which a nominated $\mathrm{S}$ is an SPSF from $\widehat{\Sigma}$ to $\Sigma$.

The class of continuous-time nonlinear stochastic hybrid systems is defined as

$\Sigma:\left\{\begin{array}{l}\mathrm{d} \xi(t)=(A \xi(t)+B \nu(t)+D w(t)+E \varphi(F \xi(t))+\mathbf{b}) \mathrm{d} t+G \mathrm{~d}_{\mathbb{W}}+\sum_{z=1}^{\mathrm{r}} R_{z} \mathrm{~d} \mathbb{P}_{t}^{z}, \\ \zeta(t)=C \xi(t),\end{array}\right.$

where $A \in \mathbb{R}^{n \times n}, B \in \mathbb{R}^{n \times m}, C \in \mathbb{R}^{q \times n}, D \in \mathbb{R}^{n \times p}, E \in \mathbb{R}^{n \times 1}, F \in \mathbb{R}^{1 \times n}$, $G \in \mathbb{R}^{n \times 1}, \mathbf{b} \in \mathbb{R}^{n \times 1}$, and $R_{i} \in \mathbb{R}^{n \times 1}, \forall z \in[1 ; \cdots ; \mathbf{r}]$. We use the tuple

$$
\Sigma=(A, B, C, D, E, F, G, \mathbf{b}, R, \varphi, \lambda)
$$

where $R=\left\{R_{1}, \ldots, R_{\mathrm{r}}\right\}, \lambda=\left\{\lambda_{1}, \ldots, \lambda_{\mathrm{r}}\right\}$ with $\lambda_{z}$ as the rates of Poisson processes $\mathbb{P}_{t}^{z}$, to refer to the class of stochastic hybrid systems in 21$]$. The discrete- 
time infinite abstraction of $\Sigma$ is described by

$$
\widetilde{\Sigma}:\left\{\begin{array}{l}
\tilde{\xi}(k+1)=\tilde{\xi}(k)+\tilde{\nu}(k)+\tilde{D} \tilde{w}(k)+\tilde{R} \varsigma(k), \quad k \in \mathbb{N}, \\
\tilde{\zeta}(k)=\tilde{C} \tilde{\xi}(k),
\end{array}\right.
$$

where $\tilde{D}$ and $\tilde{R}$ are arbitrarily chosen. Our goal here is to use $\widetilde{\Sigma}$ as the time-

discretized version of $\Sigma$ in order to establish an SPSF from $\widehat{\Sigma}$ to $\Sigma$ via $\widetilde{\Sigma}$ while finding the best approximation error. Later, we show that $\tilde{R}=\mathbf{0}_{n}$ and $\tilde{D}=$ $\mathbf{0}_{n \times p}$ result in the least approximation error (cf. Remark 5.3).

Case Study (Continued). The discrete-time infinite abstraction of $\Sigma_{i}$ is given by

$$
\widetilde{\Sigma}_{i}:\left\{\begin{array}{l}
\tilde{T}_{i}(k+1)=\tilde{T}_{i}(k)+\tilde{\nu}_{i}(k), \quad k \in \mathbb{N} . \\
\tilde{\zeta}_{i}(k)=\tilde{T}_{i}(k),
\end{array}\right.
$$

Note that, as discussed in Remark 5.3, we consider here $\tilde{R}_{i}=\tilde{D}_{i}=0$ in order to have the smallest constants $\psi_{i}$ for each $S_{i}$ (which results in smaller probabilistic error).

We present the discrete-time finite abstraction of $\widetilde{\Sigma}$ as

$$
\widehat{\Sigma}:\left\{\begin{array}{l}
\hat{\xi}(k+1)=\Phi_{\tilde{\xi}}(\hat{\xi}(k)+\hat{\nu}(k)+\tilde{D} \hat{w}(k)+\tilde{R} \varsigma(k)), \quad k \in \mathbb{N}, \\
\hat{\zeta}(k)=\hat{C} \hat{\xi}(k),
\end{array}\right.
$$

where map $\Phi_{\tilde{\xi}}: \tilde{X} \rightarrow \hat{X}$ satisfies inequality (8). Now we nominate the following quadratic simulation function

$$
S(x, \hat{x})=(x-P \hat{x})^{T} M(x-P \hat{x}),
$$

where $P$ is a square matrix and $M$ is a positive-definite matrix of appropriate dimension. In order to show that the nominated $S$ in 24 is an SPSF from $\widehat{\Sigma}$ to $\Sigma$, we require the following two key assumptions on $\Sigma$.

Assumption 2. Assume that there exists a concave function $\gamma \in \mathcal{K}_{\infty}$ such that $S$ satisfies

$$
S\left(x, x^{\prime}\right)-S\left(x, x^{\prime \prime}\right) \leq \gamma\left(\left\|x^{\prime}-x^{\prime \prime}\right\|\right),
$$

for any $x, x^{\prime}, x^{\prime \prime} \in X$. 
Note that as shown in [4] and by employing the mean value theorem, Assumption 2 is always satisfied for function $S$ in (24) being restricted to a compact subset of $X \times X$.

Assumption 3. Let $\Sigma=(A, B, C, D, E, F, G, \mathbf{b}, R, \varphi, \lambda)$. Assume that for some constant $\tilde{\kappa} \in \mathbb{R}_{>0}$, there exist matrices $M \succ 0, K, P, Q, L$ and $H$ of appropriate dimensions such that the following matrix (in)equalities hold:

$$
\begin{aligned}
(A+B K)^{T} M+M(A+B K) & \preceq-\tilde{\kappa} M, \\
A P & =B Q, \\
E & =B L, \\
D & =B H .
\end{aligned}
$$

Note that there exist matrices $Q, L$, and $H$ satisfying conditions 27, 28), and (29) if and only if im $A P \subseteq \operatorname{im} B, \operatorname{im} E \subseteq \operatorname{im} B$, and $\operatorname{im} D \subseteq \operatorname{im} B$, respectively. Now, we provide another main results of the paper showing that under Assumptions 2 and 3 , function $S$ in (24) is an SPSF from $\widehat{\Sigma}$ to $\Sigma$. Theorem 5.1. Let $\Sigma=(A, B, C, D, E, F, G, \mathbf{b}, R, \varphi, \lambda)$ and $\widehat{\Sigma}$ be its discretetime finite abstraction with discretization parameter $\delta$. Suppose Assumptions 2 and 3 hold, and $\hat{C}=\tilde{C}=C P$. Then function $S$ in (24) is an SPSF from $\widehat{\Sigma}$ to $\Sigma$.

The proof of Theorem 5.1 is provided in the Appendix.

Remark 5.2. Note that we nominated the simulation function in the quadratic form as in (24) and obtained the matrix inequality condition (26). Satisfying this inequality has a necessary and sufficient condition which is stabilizability of the pair $(A, B)$. Alternatively, other forms of simulation functions can be used but the corresponding required conditions need to be obtained according to the definition of the simulation function and for example sum of squares programs 42. 
Case Study (Continued). Conditions (26)-(29) are satisfied by $M_{i}=$ $1, P_{i}=1, Q_{i}=-0.21, L_{i}=1, H_{i}=0.1$. By taking $\tau=0.1, \lambda_{i}=0.5, \pi_{i}=$ $1, \tilde{\pi}_{i}=0.99, \tilde{\delta}_{i}=0.01, \tilde{\delta}_{i}^{\prime}=1, \bar{\eta}_{i}=0.01$, function $S_{i}\left(T_{i}(k \tau), \tilde{T}_{i}(k)\right)=\left(T_{i}(k \tau)-\right.$ $\left.{ }_{425} \tilde{T}_{i}(k)\right)^{2}$ is an SPSF from $\widetilde{\Sigma}_{i}$ to $\Sigma_{i}$ satisfying condition (9) with $\alpha_{i}(s)=s^{2}, \forall s \in$ $\mathbb{R}_{\geq 0}$ and condition 10 with $\kappa_{i}=0.99, \rho_{\text {ext } i}(s)=2.04 s, \rho_{\text {int } i}(s)=7.78 \times$ $10^{-11} s^{2}, \forall s \in \mathbb{R}_{\geq 0}$, and $\psi_{i}=1.36 \times 10^{-8}$.

The functions and constants $\alpha, \rho_{\text {ext }}, \rho_{\text {int }} \in \mathcal{K}_{\infty}, 0<\kappa<1$, and $\psi \in \mathbb{R}_{>0}$ in Definition 3.1 associated with $S$ in 24 are obtained as

$$
\begin{aligned}
\alpha(s):= & \frac{\lambda_{\min }(M)}{n \lambda_{\max }\left(C^{T} C\right)} s^{2}, \quad \forall s \in \mathbb{R}_{\geq 0}, \\
\kappa:= & 1-(1-\tilde{\pi}) \bar{\kappa}, \\
\rho_{\mathrm{ext}}(s):= & (1+\tilde{\delta})\left(\frac{1}{\tilde{\pi} \bar{\kappa}}\right) \gamma\left(\left(1+\frac{1}{\bar{\eta}}\right)\left(1+\bar{\eta}^{\prime}\right)\left(1+\bar{\eta}^{\prime \prime}\right) s\right), \quad \forall s \in \mathbb{R}_{\geq 0}, \\
\rho_{\text {int }}(s):= & (1+1 / \tilde{\delta})\left(\frac{1}{\tilde{\pi} \bar{\kappa}}\right)\left(1+\tilde{\delta}^{\prime}\right) e^{-\tilde{\kappa} \tau} \tau p \pi\|\sqrt{M} D\|_{2}^{2} s^{2}, \quad \forall s \in \mathbb{R}_{\geq 0}, \\
\psi:= & (1+1 / \tilde{\delta})\left(\frac{1}{\tilde{\pi} \bar{\kappa}}\right)\left(1+1 / \tilde{\delta}^{\prime}\right)\left(e ^ { - \overline { \kappa } \tau } \tau \left(G^{T} M G+\pi\|\sqrt{M} \mathbf{b}\|_{2}^{2}\right.\right. \\
& \left.+\sum_{z=1}^{r} \lambda_{z} R_{z}^{T} M R_{z}+\pi\left\|\sqrt{M} \sum_{z=1}^{r} \lambda_{z} R_{z}\right\|_{2}^{2}\right)+\gamma((1+\bar{\eta}) \delta) \\
& \left.+\gamma\left(\left(1+\frac{1}{\bar{\eta}}\right)\left(1+\frac{1}{\bar{\eta}^{\prime}}\right) \sqrt{\operatorname{Tr}\left(\tilde{R}^{T} \tilde{R}\right)}\right)+\gamma\left(\left(1+\frac{1}{\bar{\eta}}\right)\left(1+\bar{\eta}^{\prime}\right)\left(1+\frac{1}{\bar{\eta}^{\prime \prime}}\right)\|\tilde{D}\|\|\hat{w}\|\right)\right),
\end{aligned}
$$

where $\bar{\kappa}=1-e^{-\tilde{\kappa} \tau}$, and $0<\tilde{\pi}<1$ and $\tilde{\delta}, \tilde{\delta}^{\prime}, \bar{\eta}, \bar{\eta}^{\prime}, \bar{\eta}^{\prime \prime}>0$ are chosen arbitrarily.

Note that if $\gamma$ is linear, then $\rho_{\text {ext }}$, and $\psi$ defined in 10 reduce to

$$
\begin{aligned}
\rho_{\text {ext }}(s):= & (1+\tilde{\delta})\left(\frac{1}{\tilde{\pi} \bar{\kappa}}\right) \gamma(s), \quad \forall s \in \mathbb{R}_{\geq 0}, \\
\psi:= & (1+1 / \tilde{\delta})\left(\frac{1}{\tilde{\pi} \bar{\kappa}}\right)\left(1+1 / \tilde{\delta}^{\prime}\right)\left(e ^ { - \tilde { \kappa } \tau } \tau \left(G^{T} M G+\pi\|\sqrt{M} \mathbf{b}\|_{2}^{2}\right.\right. \\
& \left.\left.+\sum_{z=1}^{r} \lambda_{z} R_{z}^{T} M R_{z}+\pi\left\|\sqrt{M} \sum_{z=1}^{r} \lambda_{z} R_{z}\right\|_{2}^{2}\right)+\gamma(\delta)+\gamma\left(\sqrt{\operatorname{Tr}\left(\tilde{R}^{T} \tilde{R}\right)}\right)+\gamma(\|\tilde{D}\|\|\hat{w}\|)\right) .
\end{aligned}
$$

Remark 5.3. Note that for the abstraction $\widetilde{\Sigma}$ in $(22), \rho_{\text {ext }}$, and $\psi$ defined 
in 10 reduce to

$$
\begin{aligned}
\rho_{\mathrm{ext}}(s):= & (1+\tilde{\delta})\left(\frac{1}{\tilde{\pi} \bar{\kappa}}\right) \gamma\left((1+\bar{\eta})\left(1+\bar{\eta}^{\prime}\right) s\right), \quad \forall s \in \mathbb{R}_{\geq 0}, \\
\psi:= & (1+1 / \tilde{\delta})\left(\frac{1}{\tilde{\pi} \bar{\kappa}}\right)\left(1+1 / \tilde{\delta}^{\prime}\right)\left(e ^ { - \tilde { \kappa } \tau } \tau \left(G^{T} M G+\pi\|\sqrt{M} \mathbf{b}\|_{2}^{2}+\sum_{z=1}^{r} \lambda_{z} R_{z}^{T} M R_{z}\right.\right. \\
& \left.\left.+\pi\left\|\sqrt{M} \sum_{z=1}^{r} \lambda_{z} R_{z}\right\|_{2}^{2}\right)+\gamma\left(\left(1+\frac{1}{\bar{\eta}}\right) \sqrt{\operatorname{Tr}\left(\tilde{R}^{T} \tilde{R}\right)}\right)+\gamma\left((1+\bar{\eta})\left(1+\frac{1}{\bar{\eta}^{\prime}}\right)\|\tilde{D}\|\|\hat{w}\|\right)\right) .
\end{aligned}
$$

Moreover, if the abstraction $\widetilde{\Sigma}$ is non-stochastic (i.e. $\tilde{R}=\mathbf{0}_{n}$ ) with $\tilde{D}=\mathbf{0}_{n \times p}$, then

$$
\begin{aligned}
\rho_{\mathrm{ext}}(s):= & (1+\tilde{\delta})\left(\frac{1}{\tilde{\pi} \bar{\kappa}}\right) \gamma(s), \quad \forall s \in \mathbb{R}_{\geq 0}, \\
\psi:= & (1+1 / \tilde{\delta})\left(\frac{1}{\tilde{\pi} \bar{\kappa}}\right)\left(1+1 / \tilde{\delta}^{\prime}\right)\left(e ^ { - \tilde { \kappa } \tau } \tau \left(G^{T} M G+\pi\|\sqrt{M} \mathbf{b}\|_{2}^{2}\right.\right. \\
& \left.+\sum_{z=1}^{r} \lambda_{z} R_{z}^{T} M R_{z}+\pi\left\|\sqrt{M} \sum_{z=1}^{r} \lambda_{z} R_{z}\right\|_{2}^{2}\right) .
\end{aligned}
$$

This means if the concrete system has some stability property, it is actually better to go with the non-stochastic infinite abstractions than the stochastic ones since the non-stochastic abstractions are closer than the stochastic versions to the concrete systems ( $c f$. the case study).

Remark 5.4. Note that not having any internal input in the abstract systems in (23) (i.e. $\tilde{D}=\mathbf{0}_{n \times p}$ ) will actually result in less approximation error. In fact, the smart choice of the interface map in (33) still ensures that the output trajectories of abstract systems follow those of the original ones with a quantified probabilistic error bound which gets smaller if $\tilde{D}=\mathbf{0}_{n \times p}$.

Case Study (Continued). Now we proceed with checking the small-gain condition (18) that is required for the compositionality result. By taking $\bar{\sigma}_{i}(s)=$ ${ }_{440} s, \forall i \in\{1, \ldots, n\}$, condition (18) and as a result condition (19) are always satisfied. Hence, $V(T(k \tau), \hat{T}(k))=\max _{i}\left(T_{i}(k \tau)-\hat{T}_{i}(k)\right)^{2}$ is an SSF from $\widetilde{\Sigma}$ to $\Sigma$ satisfying conditions (11) and 12 with $\alpha(s)=s^{2}, \forall s \in \mathbb{R}_{\geq 0}, \kappa=0.99$, $\rho_{\text {ext }}(s)=2.04 s, \forall s \in \mathbb{R}_{\geq 0}$, and $\psi=1.36 \times 10^{-8}$.

By taking the initial states of $\Sigma$ and $\widetilde{\Sigma}$ as $20.5 \mathbb{1}_{1000}$, and utilizing Theorem 3.6 . one can guarantee that the distance between outputs of $\Sigma$ and $\widetilde{\Sigma}$ will 
not exceed $\varepsilon=0.5$ during the time horizon $T_{d}=12$ with probability at least $91 \%$, i.e.,

$$
\mathbb{P}(\|\zeta(k \tau)-\tilde{\zeta}(k)\| \leq 0.5, \forall k \in[0,12]) \geq 0.91
$$

We now synthesize a controller for $\Sigma$ via its discrete-time abstraction $\widetilde{\Sigma}$ such that the controller keeps the temperature of each room in a safe set [20,21]. The idea here is to design a local controller for the abstract subsystem $\widetilde{\Sigma}_{i}$, and then refine it back to subsystem $\Sigma_{i}$ via the interface function. We employ the software tool SCOTS 43] on a machine with Linux Ubuntu (Intel i7@3.6GHz CPU and 16 GB of RAM) to synthesize controllers for $\widetilde{\Sigma}_{i}$ maintaining the temperature of each room in the comfort zone $[20,21]$. The required memory usage and computation time for synthesizing controllers for each room are respectively $184 \mathrm{MB}$ and 70 seconds. Closed-loop state trajectories of a representative room with different noise realizations in a network of 1000 rooms are illustrated in Figure 5. Furthermore, several realizations of the norm of error between outputs of $\Sigma$ and $\widetilde{\Sigma}$ are illustrated in Figure 6 . In order to provide more practical analysis on the proposed probabilistic bound, we also run Monte Carlo simulation for 10000 runs. In this case, one can statistically guarantee that the distance between outputs of $\Sigma$ and $\widetilde{\Sigma}$ is always less than or equal to 0.24 with the same probability (i.e., at least 91\%). This issue is expected and the reason is due to the conservative nature of simulation functions, but with the gain of providing formal guarantees on the probabilistic distance between output trajectories rather than empirical ones. Note that we intentionally dropped the noise and instead used SCOTS 43]. The reason is because we formally showed that if the concrete system has some nice stability property and the two systems are in continuous-time and discrete-time domains, it is actually better to construct and employ the non-stochastic abstraction (as discussed in Remark 5.3).

\subsection{Analysis on Probabilistic Closeness Guarantee}

In order to have a practical analysis on the probabilistic closeness guarantee, we provide Table 1 in which we discuss the proposed closeness guarantees for different values of time horizon, closeness precision, diffusion and reset terms. 


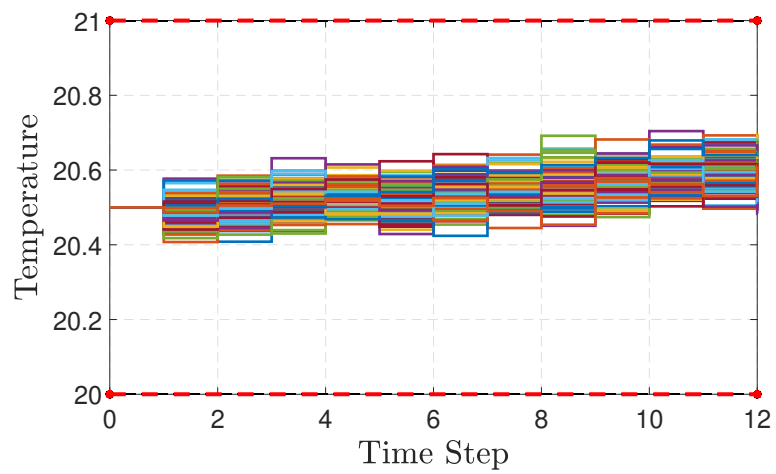

Figure 5: Closed loop state trajectories of a representative room with different noise realizations in a network of 1000 rooms, for $T_{d}=12$.

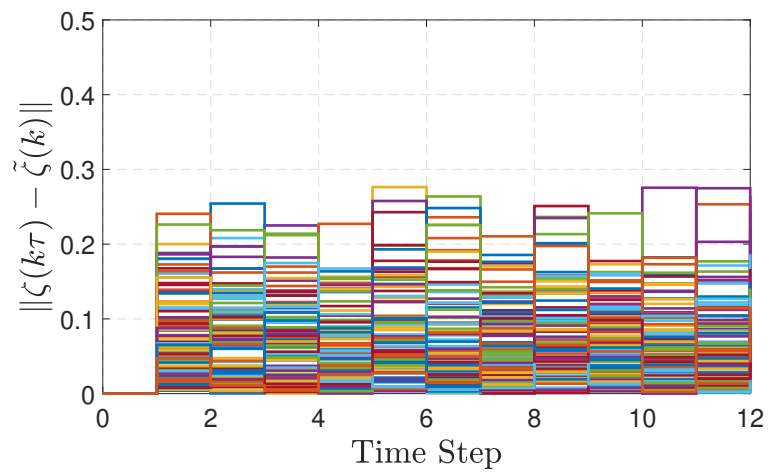

Figure 6: Several realizations of the norm of the error between the outputs of $\Sigma$ and of $\widetilde{\Sigma}$, i.e. $\|\zeta(k \tau)-\tilde{\zeta}(k)\|$, for $T_{d}=12$. 
Table 1: Probabilistic error bound proposed in 13 based on $T_{d}, \varepsilon, G$ and $R$.

\begin{tabular}{l|l|l|l|l|l|l} 
Time horizon $T_{d}$ & 5 & 10 & 15 & 20 & 30 & 40 \\
\hline Probabilistic closeness & $96 \%$ & $92 \%$ & $88 \%$ & $85 \%$ & $78 \%$ & $71 \%$ \\
\hline Precision $\varepsilon$ & 0.1 & 0.3 & 0.5 & 0.7 & 0.9 & 1.1 \\
\hline Probabilistic closeness & $6 \%$ & $75 \%$ & $91 \%$ & $95 \%$ & $97 \%$ & $98 \%$ \\
\hline Diffusion term $G$ & 0.1 & 0.3 & 0.5 & 0.7 & 0.9 & 1.1 \\
\hline Probabilistic closeness & $63 \%$ & $59 \%$ & $53 \%$ & $45 \%$ & $35 \%$ & $27 \%$ \\
\hline Reset term $R$ & 0.1 & 0.3 & 0.5 & 0.7 & 0.9 & 1.1 \\
\hline Probabilistic closeness & $75 \%$ & $68 \%$ & $53 \%$ & $37 \%$ & $23 \%$ & $12 \%$ \\
\hline
\end{tabular}

We fixed the employed parameters in the case study and computed the closeness for different ranges of $T_{d}, G, R, \varepsilon$. We have also fixed $\tau=0.03$ for computing the probabilistic bound for $G, R$. As seen, the probabilistic closeness guarantee is improved by either decreasing $T_{d}, G, R$ or increasing $\varepsilon$. Note that constant $\psi$ 475 in 13 is formulated based on diffusion and reset terms as in 30 .

\section{Discussion}

In this paper, we proposed a compositional framework for the construction of discrete-time finite MDPs of continuous-time stochastic hybrid systems. We first introduced notions of stochastic pseudo-simulation and simulation functions for quantifying the probabilistic error between outputs of the original continuoustime stochastic hybrid systems and those of their discrete-time (finite or infinite) abstractions with and without internal inputs, respectively. We also derived sufficient small-gain conditions for the compositional quantification of the probabilistic distance between the interconnection of original continuous-time stochastic hybrid systems and their discrete-time abstractions. We then constructed finite abstractions together with their corresponding stochastic pseudosimulation functions for a special class of nonlinear stochastic hybrid systems. We illustrated the effectiveness of the proposed approaches by applying our 
results to the temperature regulation in a circular building and constructing compositionally a discrete-time abstraction from its original continuous-time dynamic in a network containing 1000 rooms. We employed the constructed discrete-time abstraction as a substitute to compositionally synthesize policies regulating the temperature of each room for a bounded time horizon. Providing a construction framework of finite MDPs for general nonlinear stochastic hybrid systems is still open as a potential direction for a future work.

\section{Acknowledgment}

The authors would like to thank Abolfazl Lavaei for the fruitful discussions and helpful comments.

[1] A. Pnueli, "The temporal logic of programs," in Proceedings of the 18th IEEE Annual Symposium on Foundations of Computer Science, 1977, pp. $46-57$.

[2] C. Baier, J.-P. Katoen, and K. G. Larsen, Principles of model checking. MIT press, 2008.

[3] L. Laurenti, A. Abate, L. Bortolussi, L. Cardelli, M. Ceska, and M. Kwiatkowska, "Reachability computation for switching diffusions: Finite abstractions with certifiable and tuneable precision," in Proceedings of the 20th ACM International Conference on Hybrid Systems: Computation and Control, 2017, pp. 55-64.

[4] M. Zamani, P. Mohajerin Esfahani, R. Majumdar, A. Abate, and J. Lygeros, "Symbolic control of stochastic systems via approximately bisimilar finite abstractions," IEEE Transactions on Automatic Control, vol. 59, no. 12, pp. 3135-3150, 2014.

[5] M. Zamani and A. Abate, "Approximately bisimilar symbolic models for randomly switched stochastic systems," Systems \& Control Letters, vol. 69, pp. 38-46, 2014. 
[6] M. Zamani, A. Abate, and A. Girard, "Symbolic models for stochastic switched systems: A discretization and a discretization-free approach," $A u$ tomatica, vol. 55, pp. 183-196, 2015.

[7] A. A. Julius and G. J. Pappas, "Approximations of stochastic hybrid systems," IEEE Transactions on Automatic Control, vol. 54, no. 6, pp. 11931203, 2009.

[8] M. Zamani, M. Rungger, and P. Mohajerin Esfahani, "Approximations of stochastic hybrid systems: A compositional approach," IEEE Transactions on Automatic Control, vol. 62, no. 6, pp. 2838-2853, 2017.

[9] A. Abate, M. Prandini, J. Lygeros, and S. Sastry, "Probabilistic reachability and safety for controlled discrete-time stochastic hybrid systems," Automatica, vol. 44, no. 11, pp. 2724-2734, 2008.

[10] S. Soudjani and A. Abate, "Adaptive and sequential gridding procedures for the abstraction and verification of stochastic processes," SIAM Journal on Applied Dynamical Systems, vol. 12, no. 2, pp. 921-956, 2013.

[11] S. Soudjani, "Formal abstractions for automated verification and synthesis of stochastic systems," Ph.D. dissertation, Technische Universiteit Delft, The Netherlands, 2014.

[12] S. Soudjani, C. Gevaerts, and A. Abate, "FAUST": Formal abstractions of uncountable-state stochastic processes," in TACAS'15, ser. Lecture Notes in Computer Science. Springer, 2015, vol. 9035, pp. 272-286.

[13] N. Cauchi and A. Abate, "StocHy: Automated verification and synthesis of stochastic processes," in Proceedings of the International Conference on Tools and Algorithms for the Construction and Analysis of Systems. Springer, 2019, pp. 247-264.

[14] I. Tkachev, A. Mereacre, J.-P. Katoen, and A. Abate, "Quantitative automata-based controller synthesis for non-autonomous stochastic hybrid 
systems," in Proceedings of the 16th ACM International Conference on Hybrid Systems: Computation and Control, 2013, pp. 293-302.

[15] M. Kamgarpour, S. Summers, and J. Lygeros, "Control design for specifications on stochastic hybrid systems," in Proceedings of the 16th International Conference on Hybrid Systems: Computation and Control, ser. HSCC '13. New York, NY, USA: ACM, 2013, pp. 303-312.

[16] A. Lavaei, S. Soudjani, R. Majumdar, and M. Zamani, "Compositional abstractions of interconnected discrete-time stochastic control systems," in Proceedings of the 56th IEEE Conference on Decision and Control, 35513556, 2017.

[17] S. Soudjani, A. Abate, and R. Majumdar, "Dynamic Bayesian networks for formal verification of structured stochastic processes," Acta Informatica, vol. 54, no. 2, pp. 217-242, Mar 2017.

[18] A. Lavaei, S. Soudjani, and M. Zamani, "From dissipativity theory to compositional construction of finite Markov decision processes," in Proceedings of the 21st ACM International Conference on Hybrid Systems: Computation and Control, 2018, pp. 21-30.

[19] K. Mallik, S. Soudjani, A. Schmuck, and R. Majumdar, "Compositional construction of finite state abstractions for stochastic control systems," in 2017 IEEE 56th Annual Conference on Decision and Control (CDC), Dec 2017, pp. 550-557.

[20] S. Haesaert, S. Soudjani, and A. Abate, "Verification of general Markov decision processes by approximate similarity relations and policy refinement," in SIAM Journal on Control and Optimization, vol. 55, no. 4, 2017, pp. 2333-2367.

[21] A. Lavaei, S. Soudjani, and M. Zamani, "Compositional abstraction-based synthesis of general MDPs via approximate probabilistic relations," arXiv: 1906.02930, June 2019. 
[22] G. Pola, A. Girard, and P. Tabuada, "Approximately bisimilar symbolic models for nonlinear control systems," Automatica, vol. 44, no. 10, pp. 2508-2516, 2008.

[23] A. Girard, G. Pola, and P. Tabuada, "Approximately bisimilar symbolic models for incrementally stable switched systems," IEEE Transactions on Automatic Control, vol. 55, no. 1, pp. 116-126, 2009.

[24] M. Zamani, G. Pola, M. Mazo, and P. Tabuada, "Symbolic models for nonlinear control systems without stability assumptions," IEEE Transactions on Automatic Control, vol. 57, no. 7, pp. 1804-1809, 2011.

[25] A. Girard and G. J. Pappas, "Hierarchical control system design using approximate simulation," Automatica, vol. 45, no. 2, pp. 566-571, 2009.

[26] R. H. Gielen, R. M. Hermans, M. Lazar, and A. R. Teel, "On the application of the small-gain theorem to the stability analysis of large-scale power systems with delay," in Proceedings of the American Control Conference (ACC), 2012, pp. 1476-1481.

[27] D. Liberzon, D. Nešić, and A. R. Teel, "Small-gain theorems of lasalle type for hybrid systems," in Proceedings of the 51st IEEE Conference on Decision and Control (CDC), 2012, pp. 6825-6830.

[28] — , "Lyapunov-based small-gain theorems for hybrid systems," IEEE Transactions on Automatic control, vol. 59, no. 6, pp. 1395-1410, 2014.

[29] D. Tolić, R. G. Sanfelice, and R. Fierro, "Self-triggering in nonlinear systems: A small gain theorem approach," in Proceedings of the 20th Mediterranean Conference on Control \& Automation (MED), 2012, pp. 941-947.

[30] D. Tolic, R. G. Sanfelice, and R. Fierro, "Input-output triggered control via the small gain theorem and switched systems modeling," Proceedings of the International Journal of Robust and Nonlinear Control, pp. 1-27, 2014. 
[31] A. Nejati, S. Soudjani, and M. Zamani, "Abstraction-based synthesis of continuous-time stochastic control systems," in Proceedings of the 18th European Control Conference, 2019, pp. 3212-3217.

[38] E. B. Dynkin, "Markov processes." Springer, 1965, pp. 77-104.

[39] B. S. Rüffer, "Monotone inequalities, dynamical systems, and paths in the positive orthant of Euclidean n-space," Positivity, vol. 14, no. 2, pp. 257-

[32] B. K. Øksendal and A. Sulem, Applied stochastic control of jump diffusions. Springer, 2005, vol. 498.

[33] R. Goedel, R. G. Sanfelice, and A. R. Teel, "Hybrid dynamical systems: modeling stability, and robustness," 2012.

[34] A. Girard, G. Gössler, and S. Mouelhi, "Safety controller synthesis for incrementally stable switched systems using multiscale symbolic models," IEEE Transactions on Automatic Control, vol. 61, no. 6, pp. 1537-1549, 2016.

[35] S. Haesaert, S. Soudjani, and A. Abate, "Verification of general Markov decision processes by approximate similarity relations and policy refinement," SIAM Journal on Control and Optimization, vol. 55, no. 4, pp. 2333-2367, 2017.

[36] D. P. Bertsekas and S. E. Shreve, Stochastic optimal control: The discretetime case. Athena Scientific, 1996.

[37] S. Soudjani, A. Abate, and R. Majumdar, "Dynamic Bayesian networks as formal abstractions of structured stochastic processes," in Proceedings of the 26th International Conference on Concurrency Theory, 2015, pp. 1-14. $283,2010$.

[40] S. Dashkovskiy, B. S. Rüffer, and F. R. Wirth, "An ISS small gain theorem for general networks," Mathematics of Control, Signals, and Systems (MCSS), vol. 19, no. 2, pp. 93-122, 2007. 
[41] S. N. Dashkovskiy, B. S. Rüffer, and F. R. Wirth, "Small gain theorems for large scale systems and construction of ISS Lyapunov functions," SIAM Journal on Control and Optimization, vol. 48, no. 6, pp. 4089-4118, 2010.

[42] A. Papachristodoulou, J. Anderson, G. Valmorbida, S. Prajna, P. Seiler, and P. Parrilo, "SOSTOOLS version 3.00 sum of squares optimization toolbox for MATLAB," arXiv:1310.4716, 2013.

[43] M. Rungger and M. Zamani, "SCOTS: A tool for the synthesis of symbolic controllers," in Proceedings of the 19th ACM International Conference on Hybrid Systems: Computation and Control, 2016, pp. 99-104.

[44] W. H. Young, "On classes of summable functions and their Fourier series," Proceedings of the Royal Society of London A: Mathematical, Physical and Engineering Sciences, vol. 87, no. 594, pp. 225-229, 1912.

[45] T. H. Gronwall, "Note on the derivatives with respect to a parameter of the solutions of a system of differential equations," Annals of Mathematics, pp. 292-296, 1919.

[46] A. Swikir, A. Girard, and M. Zamani, "From dissipativity theory to compositional synthesis of symbolic models," in Proceedings of the 4 th Indian Control Conference (ICC), 2018, pp. 30-35.

\section{Appendix}

Proof of Theorem 4.6. We first show that SSF $V$ in 20 satisfies the inequality 11 for some $\mathcal{K}_{\infty}$ function $\alpha$. For any $x=\left[x_{1} ; \ldots ; x_{N}\right] \in X$ and $\hat{x}=\left[\hat{x}_{1} ; \ldots ; \hat{x}_{N}\right] \in \hat{X}$, one gets:

$$
\begin{aligned}
\|h(x)-\hat{h}(\hat{x})\| & =\max _{i}\left\{\left\|h_{i i}\left(x_{i}\right)-\hat{h}_{i i}\left(\hat{x}_{i}\right)\right\|\right\} \leq \max _{i}\left\{\left\|h_{i}\left(x_{i}\right)-\hat{h}_{i}\left(\hat{x}_{i}\right)\right\|\right\} \\
& \leq \max _{i}\left\{\alpha_{i}^{-1}\left(S_{i}\left(x_{i}, \hat{x}_{i}\right)\right)\right\}=\beta\left(\max _{i}\left\{\bar{\sigma}_{i}^{-1}\left(S_{i}\left(x_{i}, \hat{x}_{i}\right)\right)\right\}\right)=\beta(V(x, \hat{x}))
\end{aligned}
$$

where $\beta(s)=\max _{i}\left\{\alpha_{i}^{-1} \circ \bar{\sigma}_{i}(s)\right\}$ for all $s \in \mathbb{R}_{\geq 0}$, which is a $\mathcal{K}_{\infty}$ function and (11) holds with $\alpha=\beta^{-1}$. 
We continue with showing (12), as well. Let $\kappa(s)=\max _{i, j}\left\{\bar{\sigma}_{i}^{-1} \circ \kappa_{i j} \circ \bar{\sigma}_{j}(s)\right\}$. It follows from 19 that $\kappa<\mathcal{I}_{d}$. Since $\max _{i} \bar{\sigma}_{i}^{-1}$ is concave, one can readily acquire the chain of inequalities in (31) using Jensen's inequality, inequality (17), and by defining $\rho_{\text {ext }}(\cdot)$, and $\psi$ as

$$
\begin{aligned}
\rho_{\mathrm{ext}}(s) & :=\left\{\begin{array}{l}
\max _{i}\left\{\bar{\sigma}_{i}^{-1} \circ \rho_{\mathrm{ext} i}\left(s_{i}\right)\right\}, \\
\text { s.t. } \quad s_{i} \geq 0,\left\|\left[s_{1} ; \ldots ; s_{N}\right]\right\|=s,
\end{array}\right. \\
\psi & :=\max _{i} \bar{\sigma}_{i}^{-1}\left(\Lambda_{i}\right),
\end{aligned}
$$

${ }_{645}$ where $\Lambda_{i}:=\left(\mathcal{I}_{d}+\tilde{\delta}_{f}^{-1}\right) \circ\left(\rho_{\text {int } i} \circ \bar{\lambda} \circ\left(\bar{\lambda}-\mathcal{I}_{d}\right)^{-1}\left(\max _{j, j \neq i}\left\{\mu_{j i}\right\}\right)+\psi_{i}\right)$. Hence, $V$ is an SSF from $\widehat{\Sigma}$ to $\Sigma$ which completes the proof.

Remark 8.1. Note that to show Theorem 4.6, we have employed the following inequalities:

$$
\left\{\begin{array}{l}
\rho_{\mathrm{int}}(a+b) \leq \rho_{\mathrm{int}} \circ \bar{\lambda}(a)+\rho_{\mathrm{int}} \circ \bar{\lambda} \circ\left(\bar{\lambda}-\mathcal{I}_{d}\right)^{-1}(b), \\
a+b \leq \max \left\{\left(\mathcal{I}_{d}+\tilde{\delta}_{f}\right)(a),\left(\mathcal{I}_{d}+\tilde{\delta}_{f}^{-1}\right)(b)\right\},
\end{array}\right.
$$

for any $a, b \in \mathbb{R}_{\geq 0}$, where $\rho_{\text {int }}, \tilde{\delta}_{f}, \bar{\lambda},\left(\bar{\lambda}-\mathcal{I}_{d}\right) \in \mathcal{K}_{\infty}$.

Proof of Theorem 5.1: Since $\hat{C}=C P$, we have $\|C x-\hat{C} \hat{x}\|^{2} \leq n \lambda_{\max }\left(C^{T} C\right)$ $\|x-P \hat{x}\|^{2}$, and similarly $\lambda_{\min }(M)\|x-P \hat{x}\|^{2} \leq(x-P \hat{x})^{T} M(x-P \hat{x})$. One can readily verify that $\frac{\lambda_{\min }(M)}{n \lambda_{\max }\left(C^{T} C\right)}\|C x-\hat{C} \hat{x}\|^{2} \leq S(x, \hat{x})$ holds $\forall x, \forall \hat{x}$, implying that inequality (9) holds with $\alpha(s)=\frac{\lambda_{\min }(M)}{n \lambda_{\max }\left(C^{T} C\right)} s^{2}$ for any $s \in \mathbb{R}_{\geq 0}$. We proceed with showing that the inequality 10 holds, as well. Using Assumption 2 , we have

$$
\begin{aligned}
& \mathbb{E}[S(\xi((k+1) \tau), \hat{\xi}(k+1)) \mid \xi=\xi(k \tau), \hat{\xi}=\hat{\xi}(k), \nu=\nu(k \tau), \hat{\nu}=\hat{\nu}(k), w=w(k \tau), \\
& \hat{w}=\hat{w}(k)]=\mathbb{E}[S(\xi((k+1) \tau), \hat{\xi}(k+1)) \mid \xi, \hat{\xi}, \nu, \hat{\nu}, w, \hat{w}]-\mathbb{E}[S(\xi((k+1) \tau), \hat{\xi}) \mid \xi, \\
& \hat{\xi}, \nu, \hat{\nu}, w, \hat{w}]+\mathbb{E}[S(\xi((k+1) \tau), \hat{\xi}) \mid \xi, \hat{\xi}, \nu, \hat{\nu}, w, \hat{w}] \leq \mathbb{E}[S(\xi((k+1) \tau), \hat{\xi}) \mid \xi, \hat{\xi}, \\
& \nu, \hat{\nu}, w, \hat{w}]+\mathbb{E}[\gamma(\|\hat{\xi}(k+1)-\hat{\xi}\|) \mid \hat{\xi}, \hat{\nu}, \hat{w}] .
\end{aligned}
$$

Now by employing Dynkin's formula 38, one obtains 


$$
\begin{aligned}
& \mathbb{E}[V(\xi((k+1) \tau), \hat{\xi}(k+1)) \mid \xi, \hat{\xi}, \nu, \hat{\nu}] \\
& =\mathbb{E}\left[\max _{i}\left\{\bar{\sigma}_{i}^{-1}\left(S_{i}\left(\xi_{i}((k+1) \tau), \hat{\xi}_{i}(k+1)\right)\right)\right\} \mid \xi, \hat{\xi}, \nu, \hat{\nu}\right] \\
& \leq \max _{i}\left\{\bar{\sigma}_{i}^{-1}\left(\mathbb{E}\left[S_{i}\left(\xi_{i}((k+1) \tau), \hat{\xi}_{i}(k+1)\right) \mid \xi, \hat{\xi}, \nu, \hat{\nu}\right]\right)\right\} \\
& =\max _{i}\left\{\bar{\sigma}_{i}^{-1}\left(\mathbb{E}\left[S_{i}\left(\xi_{i}((k+1) \tau), \hat{\xi}_{i}(k+1)\right) \mid \xi_{i}, \hat{\xi}_{i}, \nu_{i}, \hat{\nu}_{i}\right]\right)\right\} \\
& \leq \max _{i}\left\{\bar{\sigma}_{i}^{-1}\left(\max \left\{\kappa_{i}\left(S_{i}\left(x_{i}, \hat{x}_{i}\right)\right), \rho_{\mathrm{int} i}\left(\left\|w_{i}-\hat{w}_{i}\right\|\right), \rho_{\mathrm{ext} i}\left(\left\|\hat{\nu}_{i}\right\|\right), \psi_{i}\right\}\right)\right\} \\
& =\max _{i}\left\{\bar{\sigma}_{i}^{-1}\left(\max \left\{\kappa_{i}\left(S_{i}\left(x_{i}, \hat{x}_{i}\right)\right), \rho_{\operatorname{int} i}\left(\max _{j, j \neq i}\left\{\left\|w_{i j}-\hat{w}_{i j}\right\|\right\}\right), \rho_{\operatorname{ext} i}\left(\left\|\hat{\nu}_{i}\right\|\right), \psi_{i}\right\}\right)\right\} \\
& =\max _{i}\left\{\overline { \sigma } _ { i } ^ { - 1 } \left(\operatorname { m a x } \left\{\kappa_{i}\left(S_{i}\left(x_{i}, \hat{x}_{i}\right)\right), \rho_{\mathrm{inti}}\left(\max _{j, j \neq i}\left\{\left\|y_{j i}-\hat{y}_{j i}+\hat{y}_{j i}-\Phi_{\tilde{w}_{i j}}\left(\hat{y}_{j i}\right)\right\|\right\}\right),\right.\right.\right. \\
& \left.\left.\left.\rho_{\text {ext } i}\left(\left\|\hat{\nu}_{i}\right\|\right), \psi_{i}\right\}\right)\right\} \\
& \leq \max _{i}\left\{\overline { \sigma } _ { i } ^ { - 1 } \left(\operatorname { m a x } \left\{\kappa_{i}\left(S_{i}\left(x_{i}, \hat{x}_{i}\right)\right), \rho_{\operatorname{int} i}\left(\max _{j, j \neq i}\left\{\left\|h_{j}\left(x_{j}\right)-\hat{h}_{j}\left(\hat{x}_{j}\right)\right\|+\left\|\hat{y}_{j i}-\Phi_{\tilde{w}_{i j}}\left(\hat{y}_{j i}\right)\right\|\right\}\right),\right.\right.\right. \\
& \left.\left.\left.\rho_{\text {ext } i}\left(\left\|\hat{\nu}_{i}\right\|\right), \psi_{i}\right\}\right)\right\} \\
& \leq \max _{i}\left\{\bar{\sigma}_{i}^{-1}\left(\max \left\{\kappa_{i}\left(S_{i}\left(x_{i}, \hat{x}_{i}\right)\right), \rho_{\mathrm{int} i}\left(\max _{j, j \neq i}\left\{\alpha_{j}^{-1}\left(S_{j}\left(x_{j}, \hat{x}_{j}\right)\right)+\mu_{j i}\right\}\right), \rho_{\mathrm{ext} i}\left(\left\|\hat{\nu}_{i}\right\|\right), \psi_{i}\right\}\right)\right\} \\
& \leq \max _{i}\left\{\overline { \sigma } _ { i } ^ { - 1 } \left(\operatorname { m a x } \left\{\kappa_{i}\left(S_{i}\left(x_{i}, \hat{x}_{i}\right)\right), \rho_{\operatorname{int} i} \circ \bar{\lambda}\left(\max _{j, j \neq i}\left\{\alpha_{j}^{-1}\left(S_{j}\left(x_{j}, \hat{x}_{j}\right)\right)\right\}\right)\right.\right.\right. \\
& \left.\left.\left.+\rho_{\text {int } i} \circ \bar{\lambda} \circ\left(\bar{\lambda}-\mathcal{I}_{d}\right)^{-1}\left(\max _{j, j \neq i}\left\{\mu_{j i}\right\}\right), \rho_{\operatorname{ext} i}\left(\left\|\hat{\nu}_{i}\right\|\right), \psi_{i}\right\}\right)\right\} \\
& \leq \max _{i}\left\{\overline { \sigma } _ { i } ^ { - 1 } \left(\operatorname { m a x } \left\{\kappa_{i}\left(S_{i}\left(x_{i}, \hat{x}_{i}\right)\right),\left(\mathcal{I}_{d}+\tilde{\delta}_{f}\right) \circ \rho_{\mathrm{int} i} \circ \bar{\lambda}\left(\max _{j, j \neq i}\left\{\alpha_{j}^{-1}\left(S_{j}\left(x_{j}, \hat{x}_{j}\right)\right)\right\}\right),\right.\right.\right. \\
& \left.\left.\left.\rho_{\operatorname{ext} i}\left(\left\|\hat{\nu}_{i}\right\|\right), \Lambda_{i}\right\}\right)\right\} \\
& =\max _{i, j}\left\{\bar{\sigma}_{i}^{-1}\left(\max \left\{\kappa_{i j}\left(S_{j}\left(x_{j}, \hat{x}_{j}\right)\right), \rho_{\mathrm{ext} i}\left(\left\|\hat{\nu}_{i}\right\|\right), \Lambda_{i}\right\}\right)\right\} \\
& =\max _{i, j}\left\{\bar{\sigma}_{i}^{-1}\left(\max \left\{\kappa_{i j} \circ \bar{\sigma}_{j} \circ \bar{\sigma}_{j}^{-1}\left(S_{j}\left(x_{j}, \hat{x}_{j}\right)\right), \rho_{\text {ext } i}\left(\left\|\hat{\nu}_{i}\right\|\right), \Lambda_{i}\right\}\right)\right\} \\
& =\max _{i, j}\left\{\bar{\sigma}_{i}^{-1}\left(\max \left\{\kappa_{i j} \circ \bar{\sigma}_{j}(V(x, \hat{x})), \rho_{\mathrm{ext} i}\left(\left\|\hat{\nu}_{i}\right\|\right), \Lambda_{i}\right\}\right)\right\} \\
& =\max \left\{\kappa(V(x, \hat{x})), \rho_{\mathrm{ext}}(\|\hat{\nu}\|), \psi\right\} .
\end{aligned}
$$




$$
\begin{aligned}
\mathbb{E} & {[S(\xi((k+1) \tau), \hat{\xi}) \mid \xi, \hat{\xi}, \nu, \hat{\nu}, w, \hat{w}]+\mathbb{E}[\gamma(\|\hat{\xi}(k+1)-\hat{\xi}\|) \mid \hat{\xi}, \hat{\nu}, \hat{w}] } \\
& =\mathbb{E}_{\varsigma}\left[S(\xi, \hat{\xi})+\mathbb{E}\left[\int_{k \tau}^{(k+1) \tau} \mathcal{L} S(\xi(t), \hat{\xi}) \mathrm{d} t\right] \mid \hat{\xi}, \hat{\nu}, \hat{w}\right]+\mathbb{E}[\gamma(\|\hat{\xi}(k+1)-\hat{\xi}\|) \mid \hat{\xi}, \hat{\nu}, \hat{w}]
\end{aligned}
$$

Since the infinitesimal generator $\mathcal{L} S$ acting on function $S$ is defined as

$$
\begin{aligned}
\mathcal{L} S(\xi, \hat{\xi})= & \partial_{\xi} S(\xi, \hat{\xi}) f(\xi, \nu, w)+\frac{1}{2} \operatorname{Tr}\left(\sigma(\xi) \sigma(\xi)^{T} \partial_{\xi, \xi} S(\xi, \hat{\xi})\right) \\
& +\sum_{j=1}^{r} \lambda_{j}\left(S\left(\xi+\rho(\xi) e_{j}^{\mathrm{r}}, \hat{\xi}\right)-S(\xi, \hat{\xi})\right),
\end{aligned}
$$

where $e_{j}^{r}$ denotes an r-dimensional vector with 1 on the $j$-th entry and 0 elsewhere, and

$$
\partial_{\xi} S(\xi, \hat{\xi})=2(\xi(t)-P \hat{\xi})^{T} M, \quad \partial_{\xi, \xi} S(\xi, \hat{\xi})=2 M
$$

then one has

$$
\begin{aligned}
\mathbb{E}_{\varsigma} & {\left[S(\xi, \hat{\xi})+\mathbb{E}\left[\int_{k \tau}^{(k+1) \tau} \mathcal{L} S(\xi(t), \hat{\xi}) \mathrm{d} t\right] \mid \hat{\xi}, \hat{\nu}, \hat{w}\right]+\mathbb{E}[\gamma(\|\hat{\xi}(k+1)-\hat{\xi}\|) \mid \hat{\xi}, \hat{\nu}, \hat{w}] } \\
= & \mathbb{E}_{\varsigma}\left[S(\xi, \hat{\xi})+\mathbb{E}\left[\int _ { k \tau } ^ { ( k + 1 ) \tau } \left(2(\xi(t)-P \hat{\xi})^{T} M(A \xi(t)+E \varphi(F \xi(t))+B \nu(t)\right.\right.\right. \\
& \left.\left.+\mathbf{b}+D w(t))+G^{T} M G+2(\xi(t)-P \hat{\xi})^{T} M \sum_{z=1}^{r} \lambda_{z} R_{z}+\sum_{z=1}^{r} \lambda_{z} R_{z}^{T} M R_{z}\right) \mathrm{~d} t\right] \\
& \mid \hat{\xi}, \hat{\nu}, \hat{w}]+\mathbb{E}[\gamma(\|\hat{\xi}(k+1)-\hat{\xi}\|) \mid \hat{\xi}, \hat{\nu}, \hat{w}] .
\end{aligned}
$$

Given any $\xi(t), \hat{\xi}(k), w(t)$ and $\hat{w}(k)$, we choose $\nu(t)$ via the following interface function:

$$
\nu(t)=K(\xi(t)-P \hat{\xi}(k))-Q \hat{\xi}(k)-L \varphi(F \xi(t))+H(w(k \tau)-\hat{w}(k))-H w(t),
$$

where $k \tau \leq t \leq(k+1) \tau$. By employing conditions 27, 28) and 29), and 
the definition of the interface function in (33), we have

$$
\begin{aligned}
\mathbb{E}_{\varsigma} & {\left[S(\xi, \hat{\xi})+\mathbb{E}\left[\int _ { k \tau } ^ { ( k + 1 ) \tau } \left(2(\xi(t)-P \hat{\xi})^{T} M(A \xi(t)+E \varphi(F \xi(t))+B \nu(t)+\mathbf{b}+D w(t))\right.\right.\right.} \\
& \left.\left.\left.+G^{T} M G+2(\xi(t)-P \hat{\xi})^{T} M \sum_{z=1}^{r} \lambda_{z} R_{z}+\sum_{z=1}^{r} \lambda_{z} R_{z}^{T} M R_{z}\right) \mathrm{~d} t\right] \mid \hat{\xi}, \hat{\nu}, \hat{w}\right] \\
& +\mathbb{E}[\gamma(\|\hat{\xi}(k+1)-\hat{\xi}\|) \mid \hat{\xi}, \hat{\nu}, \hat{w}]=\mathbb{E}_{\varsigma}\left[S(\xi, \hat{\xi})+\mathbb{E}\left[\int _ { k \tau } ^ { ( k + 1 ) \tau } \left(2(\xi(t)-P \hat{\xi})^{T} M((A+\right.\right.\right. \\
& B K)(\xi(t)-P \hat{\xi})+\mathbf{b}+D(w-\hat{w}))+G^{T} M G+2(\xi(t)-P \hat{\xi})^{T} M \sum_{z=1}^{r} \lambda_{z} R_{z} \\
& \left.\left.\left.+\sum_{z=1}^{r} \lambda_{z} R_{z}^{T} M R_{z}\right) \mathrm{~d} t\right] \mid \hat{\xi}, \hat{\nu}, \hat{w}\right]+\mathbb{E}[\gamma(\|\hat{\xi}(k+1)-\hat{\xi}\|) \mid \hat{\xi}, \hat{\nu}, \hat{w}] .
\end{aligned}
$$

Using Young's inequality [44] as $a b \leq \frac{\pi}{2} a^{2}+\frac{1}{2 \pi} b^{2}$, for any $a, b \geq 0$ and any $\pi>0$, by employing Cauchy-Schwarz inequality and using condition (26), one has

$$
\begin{aligned}
& \mathbb{E}_{\varsigma}\left[S(\xi, \hat{\xi})+\mathbb{E}\left[\int _ { k \tau } ^ { ( k + 1 ) \tau } \left(2(\xi(t)-P \hat{\xi})^{T} M((A+B K)(\xi(t)-P \hat{\xi})+\mathbf{b}+D(w-\hat{w}))\right.\right.\right. \\
& \left.\left.\left.\quad+G^{T} M G+2(\xi(t)-P \hat{\xi})^{T} M \sum_{z=1}^{r} \lambda_{z} R_{z}+\sum_{z=1}^{r} \lambda_{z} R_{z}^{T} M R_{z}\right) \mathrm{~d} t\right] \mid \hat{\xi}, \hat{\nu}, \hat{w}\right] \\
& \quad+\mathbb{E}[\gamma(\|\hat{\xi}(k+1)-\hat{\xi}\|) \mid \hat{\xi}, \hat{\nu}, \hat{w}] \leq \mathbb{E}_{\varsigma}\left[S(\xi, \hat{\xi})+\mathbb{E}\left[\int _ { k \tau } ^ { ( k + 1 ) \tau } \left(-\tilde{\kappa} S(\xi(t), \hat{\xi})+\pi\|\sqrt{M} \mathbf{b}\|_{2}^{2}\right.\right.\right. \\
& \left.\left.\quad+\pi\|\sqrt{M} D\|_{2}^{2}\|w-\hat{w}\|_{2}^{2}+G^{T} M G+\sum_{z=1}^{r} \lambda_{z} R_{z}^{T} M R_{z}+\pi\left\|\sqrt{M} \sum_{z=1}^{r} \lambda_{z} R_{z}\right\|_{2}^{2}\right) \mathrm{~d} t\right] \\
& \mid \hat{\xi}, \hat{\nu}, \hat{w}]+\mathbb{E}[\gamma(\|\hat{\xi}(k+1)-\hat{\xi}\|) \mid \hat{\xi}, \hat{\nu}, \hat{w}]=\mathbb{E}_{\varsigma}\left[S(\xi, \hat{\xi})+\mathbb{E}\left[\int_{k \tau}^{(k+1) \tau}-\tilde{\kappa} S(\xi(t), \hat{\xi}) \mathrm{d} t\right.\right. \\
& \quad+\tau\left(\pi\|\sqrt{M} \mathbf{b}\|_{2}^{2}+\pi\|\sqrt{M} D\|_{2}^{2}\|w-\hat{w}\|_{2}^{2}+G^{T} M G+\sum_{z=1}^{r} \lambda_{z} R_{z}^{T} M R_{z}\right. \\
& \left.\left.\left.\quad+\pi\left\|\sqrt{M} \sum_{z=1}^{r} \lambda_{z} R_{z}\right\|_{2}^{2}\right)\right] \mid \hat{\xi}, \hat{\nu}, \hat{w}\right]+\mathbb{E}[\gamma(\|\hat{\xi}(k+1)-\hat{\xi}\|) \mid \hat{\xi}, \hat{\nu}, \hat{w}] .
\end{aligned}
$$


Using Gronwall inequality [45], one has

$$
\begin{aligned}
& \mathbb{E}_{\varsigma}\left[S(\xi, \hat{\xi})+\mathbb{E}\left[\int_{k \tau}^{(k+1) \tau}-\tilde{\kappa} S(\xi(t), \hat{\xi}) \mathrm{d} t+\tau\left(\pi\|\sqrt{M} \mathbf{b}\|_{2}^{2}+\pi\|\sqrt{M} D\|_{2}^{2}\|w-\hat{w}\|_{2}^{2}\right.\right.\right. \\
& \left.\left.\left.\quad+G^{T} M G+\sum_{z=1}^{r} \lambda_{z} R_{z}^{T} M R_{z}+\pi\left\|\sqrt{M} \sum_{z=1}^{r} \lambda_{z} R_{z}\right\|_{2}^{2}\right)\right] \mid \hat{\xi}, \hat{\nu}, \hat{w}\right]+\mathbb{E}[\gamma(\|\hat{\xi}(k+1)-\hat{\xi}\|) \mid \\
& \hat{\xi}, \hat{\nu}, \hat{w}] \leq \mathbb{E}_{\varsigma}\left[e^{-\tilde{\kappa} \tau} S(\xi, \hat{\xi})+\mathbb{E}\left[e ^ { - \tilde { \kappa } \tau } \tau \left(\pi\|\sqrt{M} \mathbf{b}\|_{2}^{2}+p \pi\|\sqrt{M} D\|_{2}^{2}\|w-\hat{w}\|^{2}\right.\right.\right. \\
& \left.\left.\left.\quad+G^{T} M G+\sum_{z=1}^{r} \lambda_{z} R_{z}^{T} M R_{z}+\pi\left\|\sqrt{M} \sum_{z=1}^{r} \lambda_{z} R_{z}\right\|_{2}^{2}\right)\right] \mid \hat{\xi}, \hat{\nu}, \hat{w}\right]+\mathbb{E}[\gamma(\|\hat{\xi}(k+1)-\hat{\xi}\|) \mid \\
& \hat{\xi}, \hat{\nu}, \hat{w}]=e^{-\tilde{\kappa} \tau} S(\xi, \hat{\xi})+e^{-\tilde{\kappa} \tau} \tau\left(G^{T} M G+\pi\|\sqrt{M} \mathbf{b}\|_{2}^{2}+p \pi\|\sqrt{M} D\|_{2}^{2}\|w-\hat{w}\|^{2}\right. \\
& \left.\quad+\sum_{z=1}^{r} \lambda_{z} R_{z}^{T} M R_{z}+\pi\left\|\sqrt{M} \sum_{z=1}^{r} \lambda_{z} R_{z}\right\|_{2}^{2}\right)+\mathbb{E}[\gamma(\|\hat{\xi}(k+1)-\hat{\xi}\|) \mid \hat{\xi}, \hat{\nu}, \hat{w}] .
\end{aligned}
$$
one has

$$
\begin{aligned}
& \mathbb{E}[\gamma(\|\hat{\xi}(k+1)-\hat{\xi}\|) \mid \hat{\xi}, \hat{\nu}, \hat{w}] \\
= & \mathbb{E}[\gamma(\|\hat{\xi}(k+1)-(\hat{\xi}+\hat{\nu}+\tilde{D} \hat{w}+\tilde{R} \varsigma)+(\hat{\xi}+\hat{\nu}+\tilde{D} \hat{w}+\tilde{R} \varsigma)-\hat{\xi}\|) \mid \hat{\xi}, \hat{\nu}, \hat{w}] \\
\leq & \mathbb{E}[\gamma(\delta+\|\hat{\nu}+\tilde{D} \hat{w}+\tilde{R} \varsigma\|) \mid \hat{\xi}, \hat{\nu}, \hat{w}] \\
\leq & \gamma((1+\bar{\eta}) \delta)+\mathbb{E}\left[\gamma\left(\left(1+\frac{1}{\bar{\eta}}\right)\|\hat{\nu}+\tilde{D} \hat{w}+\tilde{R} \varsigma\|\right) \mid \hat{\xi}, \hat{\nu}, \hat{w}\right] \\
\leq & \gamma((1+\bar{\eta}) \delta)+\gamma\left(\left(1+\frac{1}{\bar{\eta}}\right)\left(1+\bar{\eta}^{\prime}\right)\|\hat{\nu}+\tilde{D} \hat{w}\|\right)+\gamma\left(\left(1+\frac{1}{\bar{\eta}}\right)\left(1+\frac{1}{\bar{\eta}^{\prime}}\right) \mathbb{E}[\|\tilde{R} \varsigma\| \mid \hat{\xi}, \hat{\nu}, \hat{w}]\right) \\
\leq & \gamma((1+\bar{\eta}) \delta)+\gamma\left(\left(1+\frac{1}{\bar{\eta}}\right)\left(1+\bar{\eta}^{\prime}\right)\|\hat{\nu}+\tilde{D} \hat{w}\|\right)+\gamma\left(\left(1+\frac{1}{\bar{\eta}}\right)\left(1+\frac{1}{\bar{\eta}^{\prime}}\right) \mathbb{E}\left[\left([\tilde{R} \varsigma]^{T}[\tilde{R} \varsigma]\right)^{\frac{1}{2}} \mid \hat{\xi}, \hat{\nu}, \hat{w}\right]\right) \\
\leq & \gamma((1+\bar{\eta}) \delta)+\gamma\left(\left(1+\frac{1}{\bar{\eta}}\right)\left(1+\bar{\eta}^{\prime}\right)\left(1+\bar{\eta}^{\prime \prime}\right)\|\hat{\nu}\|\right)+\gamma\left(\left(1+\frac{1}{\bar{\eta}}\right)\left(1+\bar{\eta}^{\prime}\right)\left(1+\frac{1}{\bar{\eta}^{\prime \prime}}\right)\|\tilde{D}\|\|\hat{w}\|\right) \\
& +\gamma\left(\left(1+\frac{1}{\bar{\eta}}\right)\left(1+\frac{1}{\bar{\eta}^{\prime}}\right)\left(\mathbb{E}\left[[\tilde{R} \varsigma]^{T}[\tilde{R} \varsigma] \mid \hat{\xi}, \hat{\nu}, \hat{w}\right]\right)^{\frac{1}{2}}\right) \\
= & \gamma((1+\bar{\eta}) \delta)+\gamma\left(\left(1+\frac{1}{\bar{\eta}}\right)\left(1+\bar{\eta}^{\prime}\right)\left(1+\bar{\eta}^{\prime \prime}\right)\|\hat{\nu}\|\right)+\gamma\left(\left(1+\frac{1}{\bar{\eta}}\right)\left(1+\bar{\eta}^{\prime}\right)\left(1+\frac{1}{\bar{\eta}^{\prime \prime}}\right)\|\tilde{D}\|\|\hat{w}\|\right) \\
& +\gamma\left(\left(1+\frac{1}{\bar{\eta}}\right)\left(1+\frac{1}{\bar{\eta}^{\prime}}\right) \sqrt{\left.\operatorname{Tr}\left(\tilde{R} \tilde{R}^{T} \tilde{R}\right)\right),} \quad(34)\right.
\end{aligned}
$$

where $\bar{\eta}, \bar{\eta}^{\prime}, \bar{\eta}^{\prime \prime} \in \mathbb{R}_{>0}$. Then one can conclude that 


$$
\begin{aligned}
& \mathbb{E}[S(\xi((k+1) \tau), \hat{\xi}(k+1)) \mid \xi, \hat{\xi}, \nu, \hat{\nu}, w, \hat{w}] \\
& \quad \leq e^{-\tilde{\kappa} \tau} S(\xi, \hat{\xi})+\gamma\left(\left(1+\frac{1}{\bar{\eta}}\right)\left(1+\bar{\eta}^{\prime}\right)\left(1+\bar{\eta}^{\prime \prime}\right)\|\hat{\nu}\|\right)+e^{-\tilde{\kappa} \tau} \tau p \pi\|\sqrt{M} D\|_{2}^{2}\|w-\hat{w}\|^{2} \\
& \quad+e^{-\tilde{\kappa} \tau} \tau\left(G^{T} M G+\pi\|\sqrt{M} \mathbf{b}\|_{2}^{2}+\sum_{z=1}^{r} \lambda_{z} R_{z}^{T} M R_{z}+\pi\left\|\sqrt{M} \sum_{z=1}^{r} \lambda_{z} R_{z}\right\|_{2}^{2}\right)+\gamma((1+\bar{\eta}) \delta) \\
& \quad+\gamma\left(\left(1+\frac{1}{\bar{\eta}}\right)\left(1+\frac{1}{\bar{\eta}^{\prime}}\right) \sqrt{\operatorname{Tr}\left(\tilde{R}^{T} \tilde{R}\right)}\right)+\gamma\left(\left(1+\frac{1}{\bar{\eta}}\right)\left(1+\bar{\eta}^{\prime}\right)\left(1+\frac{1}{\bar{\eta}^{\prime \prime}}\right)\|\tilde{D}\|\|\hat{w}\|\right) . \quad(35)
\end{aligned}
$$

Using the previous inequality and by employing the similar argument as the one in [46, Theorem 1], one obtains

$$
\begin{aligned}
& \mathbb{E}[S(\xi((k+1) \tau), \hat{\xi}(k+1)) \mid \xi, \hat{\xi}, \nu, \hat{\nu}, w, \hat{w}] \\
& \quad \leq \max \left\{\kappa S(\xi, \hat{\xi}), \rho_{\text {int }}(\|w-\hat{w}\|), \rho_{\text {ext }}(\|\hat{\nu}\|), \psi\right\},
\end{aligned}
$$

which completes the proof with

$$
\begin{aligned}
\alpha(s):= & \frac{\lambda_{\min }(M)}{n \lambda_{\max }\left(C^{T} C\right)} s^{2}, \quad \forall s \in \mathbb{R}_{\geq 0}, \\
\kappa: & :=1-(1-\tilde{\pi}) \bar{\kappa}, \\
\rho_{\text {ext }}(s):= & (1+\tilde{\delta})\left(\frac{1}{\tilde{\pi} \bar{\kappa}}\right) \gamma\left(\left(1+\frac{1}{\bar{\eta}}\right)\left(1+\bar{\eta}^{\prime}\right)\left(1+\bar{\eta}^{\prime \prime}\right) s\right), \quad \forall s \in \mathbb{R}_{\geq 0}, \\
\rho_{\text {int }}(s):= & (1+1 / \tilde{\delta})\left(\frac{1}{\tilde{\pi} \bar{\kappa}}\right)\left(1+\tilde{\delta}^{\prime}\right) e^{-\tilde{\kappa} \tau} \tau p \pi\|\sqrt{M} D\|_{2}^{2} s^{2}, \quad \forall s \in \mathbb{R}_{\geq 0}, \\
\psi:= & (1+1 / \tilde{\delta})\left(\frac{1}{\tilde{\pi} \bar{\kappa}}\right)\left(1+1 / \tilde{\delta}^{\prime}\right)\left(e ^ { - \overline { \kappa } \tau } \tau \left(G^{T} M G+\pi\|\sqrt{M} \mathbf{b}\|_{2}^{2}+\sum_{z=1}^{r} \lambda_{z} R_{z}^{T} M R_{z}\right.\right. \\
& \left.+\pi\left\|\sqrt{M} \sum_{z=1}^{r} \lambda_{z} R_{z}\right\|_{2}^{2}\right)+\gamma((1+\bar{\eta}) \delta)+\gamma\left(\left(1+\frac{1}{\bar{\eta}}\right)\left(1+\frac{1}{\bar{\eta}^{\prime}}\right) \sqrt{\operatorname{Tr}\left(\tilde{R}^{T} \tilde{R}\right)}\right) \\
& \left.+\gamma\left(\left(1+\frac{1}{\bar{\eta}}\right)\left(1+\bar{\eta}^{\prime}\right)\left(1+\frac{1}{\bar{\eta}^{\prime \prime}}\right)\|\tilde{D}\|\|\hat{w}\|\right)\right),
\end{aligned}
$$

where $\bar{\kappa}=1-e^{-\tilde{\kappa} \tau}, 0<\tilde{\pi}<1$, and $\tilde{\delta}, \tilde{\delta}^{\prime}>0$.

Remark 8.2. Note that since the abstract system $\widehat{\Sigma}$ in this work is considered in discrete-time domain, then the infinitesimal generator $\mathcal{L} S(x, \hat{x})$ defined in 32 is different from the usual one that was employed in [4]. 